\title{
Patterns and distribution of presynaptic and postsynaptic elements within serial electron microscopic reconstructions of neuronal arbors from the medicinal leech Hirudo verbana
}

\author{
Jason E. Pipkin ${ }^{1,2}$, Eric A. Bushong ${ }^{3}$, Mark H. Ellisman ${ }^{3,4}$, and William B. Kristan Jr. ${ }^{2}$ \\ ${ }^{1}$ Neurosciences Graduate Program, University of California at San Diego, La Jolla, California \\ 92093 \\ ${ }^{2}$ Neurobiology Section, Division of Biological Sciences, University of California at San Diego, La \\ Jolla, California 92093 \\ ${ }^{3}$ National Center for Microscopy and Imaging Research, University of California at San Diego, La \\ Jolla, California 92093 \\ ${ }^{4}$ Department of Neurosciences, University of California at San Diego School of Medicine, La \\ Jolla, California 92093
}

\section{Abstract}

\begin{abstract}
Microscale connectomics involves the large-scale acquisition of high resolution serial electron micrographs from which neuronal arbors can be reconstructed and synapses detected. In addition to connectivity information, these datasets are also rich with structural information including vesicle types, number of postsynaptic partners at a given presynaptic site, and spatial distribution of synaptic inputs and outputs. Using serial blockface scanning electron microscopy (SBEM), we collected two volumes of serial EM data from ganglia of the medicinal leech. In the first volume, we sampled a small fraction of the neuropil belonging to an adult ganglion. From this dataset we measured the proportion of arbors that contain vesicles, the types of vesicles contained, and developed criteria to identify synapses and measure the number of apparent postsynaptic partners in apposition to presynaptic boutons. In the second dataset, we sampled an entire juvenile ganglion, which included the somata and arbors of all the neurons. We used this dataset to put our findings from mature tissue into the context of fully reconstructed arbors and to explore the spatial distribution of synaptic inputs and outputs on these arbors. We observed that some neurons segregate their arbors into input-only and mixed input/output zones, that other neurons contained
\end{abstract}

Correspondence to: Dr. Jason E. Pipkin, Department of Biology, Brandeis University, 415 South St, Volen 306, Waltham MA 02453. jason.e.pipkin@gmail.com.

CONFLICT OF INTEREST STATEMENT

The authors declare no conflicts of interest.

RESOURCES CITED

IMOD (http:/bio3d.colorado.edu/imod/, RRID: SCR_003297; Kremer et al., 1996) TrakEM2 (fiji.sc/TrakEM2, RRID: SCR_008954; Cardona et al., 2012)

ROLE OF AUTHORS

All authors had full access to all the data in the study and take responsibility for the integrity of the data and the accuracy of the data analysis. Study concept and design: JP, ME, WK. Acquisition of data: JP, EB. Analysis and interpretation of data: JP, WK. Drafting of the manuscript: JP, WK. Obtained funding: JP, WK. Administrative, technical, and material support: EB, ME. Study supervision: ME, WK. 
exclusively mixed input/output zones, and that still others contained only input zones. These results provide a groundwork for future behavioral studies.

\section{Graphical Abstract}

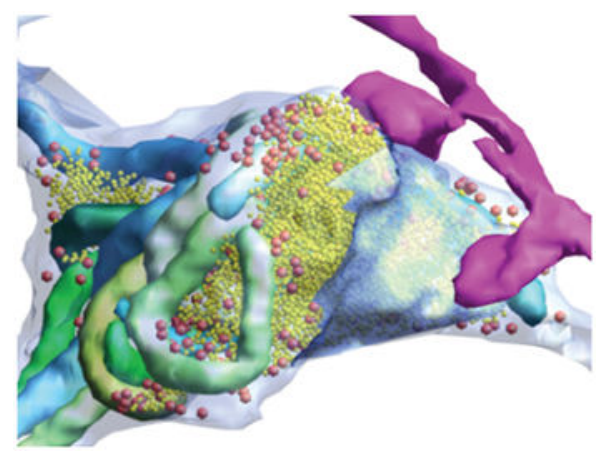

\section{Keywords}

serial blockface electron microscopy; synapses; vesicles; RRID: SCR_003297; RRID: SCR_008954

\section{INTRODUCTION}

The activity of any given neuron depends in part on the location of synaptic inputs it receives throughout its arbor. Similarly, the outputs of that neuron are linked to where it forms presynaptic specializations and what kinds of neurotransmitter-containing vesicles these possess. At a cellular and circuit level, understanding neuronal function requires an anatomical approach that reveals the details of synaptic location and composition. In the past decade, advances in serial section electron microscopy (serial EM) have enabled the collection of vast quantities of data in which entire neuronal arbors can be reconstructed while retaining the ultrastructural details necessary to identify and describe synapses (Denk and Horstmann, 2004; Briggman et al., 2011; Jarell et al., 2012; Takemura et al., 2013; Ohyama et al., 2015). While these data are typically being collected with a goal of revealing circuit-level wiring diagrams, serial EM is also an invaluable approach for revealing details like the variability of presynaptic forms among mossy fiber synapses (Wilke et al., 2013) or enabling the precise modeling of small molecule diffusion in the complex geometry of extracellular space in the hippocampal neuropil (Kinney et al., 2013).

Serial EM datasets can thus be used to describe morphological features like vesicle content, input and output locations on branches of different sizes, and number and variability of postsynaptic partners at a given presynaptic bouton. These details have been studied before at the ultrastructural level, but typically in the context of a single cell or only a small series of images (e.g. Muller and McMahan 1976; King 1976a, 1976b; Watson and Burrows, 1982, 1983, 1985). Extending the utility of the technique requires applying it to systems where multiple neuronal arbors can be identified, mapped, and annotated in their entirety. These systems can be found in the small invertebrate preparations like the ganglia of leeches, crustaceans, mollusks, and insects, which have long been favored for their physiological 
accessibility. Neurons in these systems are typically large and identifiable from preparation to preparation on the basis of their electrophysiological and morphological properties. The potential to discern ultrastructural information within a physiologically accessible system led us to apply serial EM to the leech ganglion.

The nervous system of the medicinal leech Hirudo verbana is composed of a chain of homologous ganglia linked by three connectives. Each ganglion comprises an outer layer of large neuronal somata (20-70 $\mu \mathrm{m}$ in diameter), each of which extends its primary process into an inner region of dense neuropil. Within the neuropil, neurons form profusely branched arbors that span large areas and synapse with other arbors (e.g. Muller and McMahan, 1976; DeRiemer and Macagno, 1981; Fan et al., 2005).

The outer layer of somata has been well-characterized ultrastructurally (Gray and Guillery, 1963; Coggeshall and Fawcett, 1964). Similar to other invertebrate ganglia (Coggeshall, 1967; King 1976a, 1976b; Kilman and Marder, 1996), all the neurons are monopolar and feature a central nucleus surrounded by numerous mitochondria and an elaborate network of endoplasmic reticulum that spans the entirety of the cytoplasm. A few somata contain numerous dense-core vesicles while most do not (Coggeshall and Fawcett, 1964). In at least two neurons, the large serotonergic Retzius cells, these vesicles can be released directly from the somata (Trueta et al., 2012). Dense-core vesicles are also observed in the processes of the nociceptive $\mathrm{N}$ cell that closely wrap around the somata and primary neurite of sensory neurons in adjacent ganglia (French and Muller, 1986). All the intracellular features of the somata are bounded by a plasma membrane with invaginations frequently filled by processes of glial cells that reside amongst the neurons (Gray and Guillery, 1963; Coggeshall and Fawcett, 1964).

To date, ultrastructural interrogation of the leech neuropil has been limited to what can be observed in single sections or a limited number of serial sections. Muller and McMahan (1976) used serial section transmission electron microscopy (ssTEM) to describe the general structural arrangement leech synapses as comprising a large presynaptic varicosity in apposition to several indenting postsynaptic processes. Intracellularly in the presynaptic profile, a small tuft of electron dense material would also be visible at sites where vesicles congregated near the membrane (Muller and McMahan, 1976); this is similar to that observed in molluscan and crustacean ganglia (e.g. Bailey et al., 1979; Kilman and Marder, 1996), but less dramatic than the T-bar associated with presynaptic elements in flies (Trujillo-Cenoz, 1965; Meinertzhagen, 1996). In all of these systems, the most common arrangement of synaptic elements is dyadic: two postsynaptic processes lie in apposition to each of the release sites on a larger presynaptic profile (Muller and McMahan, 1976; King, 1976a, 1976b; Bailey et al., 1979; Watson and Burrows, 1982; Meinertzhagen, 1996).

To link neuropilar structure and synapses to known cells, past efforts have employed chemical labeling approaches (e.g. somatic injection with horseradish peroxidase that is subsequently reacted with diaminobenzidine to form an osmiophilic product) that render the cytoplasm of that cell's processes differentiable from others (Muller and McMahan, 1976; King, 1976a, 1976b; Muller and Carbonetto, 1979; Watson and Burrows, 1982, 1983, 1985; Granzow et al., 1985; Wadepuhl et al., 1990). While useful for many applications, these 
stains can obscure and occasionally disrupt intracellular structures like synaptic vesicles necessary for identifying synapses (Watson and Burrows, 1982; Granzow et al., 1985).

The aim of the present study was to extend these efforts beyond single and small series of thin sections to reveal the organization and distribution of synapses in entire neuronal arbors. We employed serial blockface scanning EM (SBEM) to generate two large volumes of leech neuropil. While SBEM results in images typically of lower resolution than that achievable via TEM, the technique has the advantages of automating the sectioning process, producing data at high throughput, and involving no section warping artifacts that can affect the ease with which successive images are aligned (for a review of serial EM techniques, see Briggman and Bock, 2012).

The first image volume we collected contains a portion of neuropil from an adult ganglion that we used to describe patterns of vesicle distribution and synaptic arrangements in mature leech tissue. The second contains an entire ganglion (somata and neuropil) from a juvenile leech. We selected the smaller juvenile leech for practical purposes: their smaller ganglia ( $\sim 60 \%$ the diameter of an adult's) enable a reasonable imaging time (in our case, six weeks). Importantly, juveniles perform many of the same behaviors studied in the adult: they swim, crawl, shorten, bend, and feed (though they do not participate in reproductive behaviors) (Reynolds et al., 1998). We use the juvenile ganglion dataset to link patterns of synaptic arrangements to known cells and to report the distribution of synaptic inputs and outputs in single cells throughout the entirety of the ganglion-contained portion of their arbors.

\section{MATERIALS AND METHODS}

\section{Animals}

We used both adult and juvenile medicinal leeches Hirudo verbana. Adult leeches were obtained from Niagara Leeches (Niagara Falls, NY) and housed in aquaria on $12 \mathrm{~h}$ daily light/dark cycle at $15-16^{\circ} \mathrm{C}$. Juvenile leeches were obtained by harvesting cocoons produced by a breeding colony of adult leeches maintained in our laboratory. Leeches were allowed to mature within the cocoons at room temperature (RT) and collected once they had emerged. We then waited two weeks to ensure full development prior to dissection. We confirmed that the juveniles lacked any embryonic features using established staging criteria (Reynolds et al., 1998). For our juvenile sample, we stained and embedded several ganglia but eventually imaged only ganglion 11 .

\section{Sample preparation}

Both adult and juvenile samples were prepared with the same protocol. We anesthetized the leeches in ice-cold leech saline $\left(4^{\circ} \mathrm{C}\right)$ containing $115 \mathrm{mM} \mathrm{NaCl}, 4 \mathrm{mM} \mathrm{KCl}, 1.8 \mathrm{mM} \mathrm{CaCl}_{2}$, $2 \mathrm{mM} \mathrm{MgCl} 2,10 \mathrm{mM}$ HEPES buffer (Nicholls and Purves, 1970). Midbody ganglia were then dissected from the nerve cord and pinned them to the bottom of a Sylgard-coated dish. The ganglia were then fixed for two hours at RT in 2\% PFA, 2.5\% glutaraldehyde, and $0.1 \mathrm{M}$ phosphate buffer. After fixation the ganglia were rinsed in $0.1 \mathrm{M}$ phosphate buffer and incubated in $2 \% \mathrm{OsO}_{4} / 1.5 \%$ potassium ferrocyanide. For this step, the samples were microwaved in a scientific microwave (Pelco 3440 MAX) three times at $800 \mathrm{~W}$ with a duty 
cycle of 40 seconds on and 40 seconds off at a measured temperature of $35^{\circ} \mathrm{C}$ and subsequently left to sit at RT for thirty minutes. Samples were then washed in $\mathrm{ddH}_{2} \mathrm{O}$ and microwaved three times at $800 \mathrm{~W}$ with a duty cycle of 2 minutes on and 2 minutes off at $30^{\circ} \mathrm{C}$. We found that this and subsequent brief microwave incubations facilitated staining penetration to the center of our samples and was necessary to gain sufficient image contrast. Samples were then incubated in 1\% thiocarbohydrazide (Electron Microscopy Sciences) and microwaved three times at $800 \mathrm{~W}$ with a 40 seconds on and 40 seconds off duty cycle at $30^{\circ} \mathrm{C}$ and subsequently left to incubate for 15 minutes RT. The samples were then washed again with the same microwave incubation as described earlier. Next, the samples were incubated in $2 \%$ aqueous $\mathrm{OsO}_{4}$ and microwaved three times at $800 \mathrm{~W}$ with a 40 seconds on and 40 seconds off duty cycle at $30^{\circ} \mathrm{C}$ and then incubated at RT for one hour. After washing, the samples were then left in $1 \%$ uranyl acetate overnight at $4^{\circ} \mathrm{C}$. The next day, samples were incubated in a lead aspartate solution prepared by dissolving $0.066 \mathrm{gm}$ of lead nitrate into $10 \mathrm{ml}$ of $0.03 \mathrm{M}$ aspartic acid with the $\mathrm{pH}$ subsequently adjusted to 5.5 using $1 \mathrm{~N} \mathrm{KOH}$. This incubation took place in a $60^{\circ} \mathrm{C}$ oven for 30 minutes. The samples were then washed and dehydrated through a series of ethanol solutions $(50 \%, 70 \%, 90 \%, 100 \%, 100 \%, 10$ minutes each) at RT and incubated in acetone. Following this, samples were infiltrated with epoxy resin by first incubating them for two hours at RT in a solution of 50\% acetone and $50 \%$ Durcupan and then overnight in $100 \%$ Durcupan. The next day, samples were transferred to a freshly prepared $100 \%$ Durcupan solution and incubated at RT for 2 hours. Samples were then incubated within a $60^{\circ} \mathrm{C}$ oven for three days. Durcupan Araldite resin was made by mixing $11.4 \mathrm{~g}$ of component $\mathrm{A}, 10 \mathrm{~g}$ of component $\mathrm{B}, 0.3 \mathrm{~g}$ of component $\mathrm{C}$, and $0.1 \mathrm{~g}$ of component $\mathrm{D}$.

\section{Imaging}

The resin-embedded ganglia were preserved within carefully-trimmed epoxy blocks. For transmission EM, thin sections were cut and mounted on copper grids (no additional staining was performed) and subsequently imaged with a JEOL 1200 TEM $(120 \mathrm{kV}, 12,000 \mathrm{x}-$ 20,000x magnification). For scanning electron microscopy, blocks were trimmed until tissue was barely exposed. For the juvenile sample, the edges of the block were trimmed until very near to the external capsule of the ganglion to reduce charging in the outer image tiles that would contain both tissue and empty plastic. Blocks were mounted onto aluminum pins to which they were adhered with conductive silver paint. The pin and block were then sputter coated with a thin layer of gold and palladium to further enhance conductivity.

We imaged two samples. The first sample of adult ganglion neuropil was acquired at high vacuum on an FEI Quanta FEG SEM equipped with a Gatan 3VIEW SBEM system. The accelerating voltage was $2.5 \mathrm{kV}$ with a dwell time of $8 \mu$ s over an $8000 \times 8000$ pixel raster. Each square pixel measured $11.7 \mathrm{~nm}$ on a side and 276 sections were cut with a thickness of $70 \mathrm{~nm}$, resulting in a total imaged volume of $96 \mu \mathrm{m} \times 96 \mu \mathrm{m} \times 17.3 \mu \mathrm{m}$. The imaged region of this sample was medially located in the posterior half of the neuropil. The second sample of the entire juvenile ganglion was imaged on a Zeiss MERLIN SEM equipped with a Gatan 3 VIEW SBEM system. We collected montages of $8000 \times 8000$ raster tiles at $5.7 \mathrm{~nm}$ pixel size. We oriented the sample so that it was imaged from the dorsal surface to the ventral surface with sectioning occurring perpendicular to the dorsal-ventral axis. Montage size thus 
varied from $1 \times 1$ to $5 \times 5$ tiles depending on how large the area of tissue was that was exposed to the surface of the block. We sectioned the block 2203 times at 50,100, or $150 \mathrm{~nm}$ thicknesses for a total z-distance of $138 \mu \mathrm{m}$. The $100 \mathrm{~nm}$ and $150 \mathrm{~nm}$ sections were taken in regions containing only cell bodies (at the top and bottom of the overall volume) as there are very few fine neuronal processes to trace here and thus imaging time could be reduced.

Similarly, we varied dwell time throughout acquisition along a range of $0.8-\mu$ s to $1.5-\mu$ s with higher dwell times used in neuropil-containing sections. During the juvenile ganglion acquisition, an unexpected and gradual reduction of contrast occurred due to the premature degradation of the filament in the electron gun. As imaging proceeded from the dorsal surface towards the ventral, we therefore focused most of our analysis and reconstruction on cells whose arbors tended to fall within the dorsal half of the ganglion.

\section{Reconstruction and Annotation}

For the adult ganglion, images were aligned, analyzed, and visualized using IMOD (http:/ bio3d.colorado.edu/imod/, RRID: SCR_003297; Kremer et al., 1996). In this dataset, we traced the full neuronal membrane of target arbors in each section and separately recorded the location of features of interest, including synapses. By tracing the membrane of a given neuronal arbor, we therefore produced a "segmentation" of this arbor from the surrounding image volume. We fully segmented 96 arbors from within this image volume. These arbors were chosen based on interesting features or connectivity with other arbors and a small number of these reconstructions are shown here (Figures 1,2, and 5). In cases where we report the segmentation of vesicles (Figure 2C, D, E) ball objects were placed where each visible vesicle was located. These balls all have the same radius, as resolution limits inherent to the data prevent us from precisely segmenting each vesicle individually.

In the juvenile ganglion volume, montages and sections were aligned in the TrakEM2 (fiji.sc/TrakEM2, RRID: SCR_008954; Cardona et al., 2012). Subsequent tracing and annotation was also performed in TrakEM2. In this volume we largely reconstructed arbors via skeletonization rather than full segmentation via membrane tracing. Locations of synaptic inputs and outputs were denoted by placing ball objects as markers on the skeletons.

All tracing, segmentation, and analysis was performed by JP. To reduce errors, the arbors of the motor neurons discussed in Table 1 and Figures 10 and 11 were reviewed at least twice. As has been previously reported, false negatives (missing branches) were far more likely errors than false positives (adding the wrong branch) (Ohyama et al., 2015).

\section{Additional Analyses}

To characterize variations in the vesicle content in neuronal arbors in our volumes, we performed a "random points analysis" wherein we randomly selected coordinates and analyzed the arbors defined by each of the coordinates. For this analysis, random numbers were generated using an online service (random.org) that corresponded to the range of pixel coordinate values within each volume $(0-8,000$ for $\mathrm{x}$ and $\mathrm{y}$ coordinates and $1-276$ for $\mathrm{z}$ coordinates in the adult volume; $0-45,000$ for $\mathrm{x}$ and $\mathrm{y}$ coordinates and $300-1750$ for $\mathrm{z}$ coordinates in the juvenile volume - slices from 1-300 and 1751-2203 were excluded as 
these largely contained only cell bodies). For each point that landed in an arbor, that arbor was then traced by eye to determine its vesicle content.

We also analyzed the relationship between presynaptic vesicle field size and number of potential postsynaptic partners in mature tissue. For this analysis (Figure 8), the vesiclecontaining arbors identified from the random points analysis in the adult volume were each traced until a presynaptic bouton was identified. We then outlined the field of small synaptic vesicles in each serial section containing vesicles in that bouton. To estimate the volume of the presynaptic vesicle field, we measured the area of the vesicle field, multiplied that area by the sectioning thickness $(70 \mathrm{~nm})$ to estimate the volume in each section, then added the volumes together. The number of postsynaptic processes opposite the presynaptic bouton at that vesicle field were also recorded, as was the presence of any additional large vesicle types. These data were analyzed using Microsoft Excel (2013).

\section{RESULTS}

\section{Adult Neuropil}

Vesicle-containing and vesicle-lacking arbors-To study basic structural patterns of synaptic connectivity and describe the anatomy of mature leech neuropil, we first collected a $96 \mu \mathrm{m} \times 96 \mu \mathrm{m} \times 17.3 \mu \mathrm{m}$ volume (about $5 \%$ of the total neuropil) from an adult ganglion. When we reconstructed neuronal arbors in this volume we found that most were both presynaptic and postsynaptic to other arbors. Some arbors, however, contained no aggregations of presynaptic vesicles and were apparently only postsynaptic to other arbors. Note that these observations are confined only to chemical synapses because gap junctions cannot be positively identified at SBEM resolution. We sampled the volume at random points, visually inspecting (but not fully reconstructing) the arbor that contained each point. When doing so, we found that in this volume 87\% (131/151) of neuronal arbors contained vesicles and the remaining $13 \%$ (20/151) did not.

The arbors that lacked vesicles branched profusely and received synaptic input from the presynaptic boutons of many other arbors. With our SBEM staining protocol, these arbors tended to have distinctly clear cytoplasm, making them stand out in higher contrast from the surrounding neuropil (Figure 1A). We reconstructed several of these vesicle-lacking arbors, revealing their extensive branching patterns (three of which are shown in Figure 1B, C). Among the three arbors shown here, there is some diversity in the thickness of major branches and number of small branches. Note that all contain numerous thin (100-250nm diameter) branches (Figure 1C); these we found often terminated in apposition to presynaptic boutons and varicosities of other arbors (see arrowhead, Figure 1A).

We defined a presynaptic bouton as a region of an arbor in which there was an aggregation of small synaptic vesicles (Figures 2-4) with a maximal diameter of $40 \mathrm{~nm}$ (because our cutting thickness of $70 \mathrm{~nm}$ was too coarse to reliably measure the size of the vesicles, we instead measured maximal diameters). In some vesicle-containing arbors, small synaptic vesicles are the only kind of vesicle present, though in other boutons they were accompanied by scattered larger vesicles that typically resided near the edges of the synaptic vesicle field (Figure 2C, D, E). In other arbors, these fields of small vesicles were surrounded by 
numerous larger dense core vesicles of varying staining intensities (Figures 5 and 6). Note that the resolution limits of our datasets preclude us from discerning fine differences in vesicle morphology. For this reason, we refer to presynaptic vesicles as "small synaptic vesicles" or "small vesicles" as we cannot further classify them (e.g. as agranular or clear).

Synapse identification and vesicle fields-Like most species, at the EM level leech synapses are identified on the basis of fine features. These include the presence of a presynaptic tuft near where vesicles approach, dock, or fuse with the presynaptic membrane, a slight widening of the synaptic cleft, and, in the leech, faintly-stained postsynaptic densities (Purves and McMahan, 1972; Muller and McMahan, 1976; Muller and Carbonetto, 1979). These fine features are associated with an overall dyadic arrangement formed by the apposition of two postsynaptic processes with each of multiple presynaptic release sites in a given presynaptic varicosity (Purves and McMahan, 1972; Muller and McMahan, 1976; Muller 1979). To work within the constraints of the resolution limits of high-throughput SBEM, we therefore developed criteria for synapse identification on the basis of the features of higher resolution TEM images that could be applied to our SBEM volumes. The most frequently observed synaptic arrangement consisted of a single presynaptic bouton lying in close apposition to several smaller clear postsynaptic processes (Figure 2). In TEM, we found docked vesicles at sites where the presynaptic membrane closely followed the conformation of the postsynaptic arbors (Figure 2A); we detected this same pattern in the lower resolution SBEM sections (Figure 2B). Our major criterion for identifying chemical synaptic sites, therefore, was a concentration of vesicles in the presynaptic neuron, some of which were close to membrane appositions of the pre- and postsynaptic neurons (Figure 2B). In addition, we describe synapses only in cases where the apposition of membranes persists for three or more consecutive sections, meaning that it is theoretically possible that we do not identify some real synapses when the majority of their synaptic appositions ran parallel to the plane of sectioning (Figure 2A, B). These criteria closely match independent efforts on the detection of synapses in an ssTEM dataset spanning the nervous system of another annelid, Platynereis dumerilii (Randel et al., 2014). However, by necessity of resolution limits, our criteria are more liberal than earlier works (Muller and McMahan, 1976; Muller 1979). We cannot discern the widening of the synaptic cleft nor very fine structures like presynaptic tufts. Therefore while our criteria likely include the majority of real synapses, they probably also include some nonsynaptic appositions.

When reconstructed in three dimensions, the presynaptic boutons appeared as enlarged varicosities linked by thinner processes (Figure 2E). Each bouton had a large volume of small synaptic vesicles whose proximity to the presynaptic membrane defined a synaptic region (dark blue membrane, Figure 2C-E). Typically, in apposition to this region were several postsynaptic processes (one of which is reconstructed in pink, Figure 2C-E). This pattern is congruent with earlier reconstructions from small series of TEM images from leech neuropil (Muller and McMahon, 1976). Notably, we often found that arbors that were postsynaptic to one bouton of a presynaptic neuron were also postsynaptic to other boutons of that same neuron (Figure 2E). The pink postsynaptic arbor shown in Figure 2E is the same as the pink vesicle-lacking arbor shown in Figure 1B, C. This pattern is consistent with prior observations that known synaptic partners form multiple synapses distributed across 
their arbors (Macagno et al., 1987). We also observed cases where one presynaptic bouton made a synapse directly onto another presynaptic bouton. These were uncommon, which is also consistent with previous findings (Purves and McMahan, 1972; Muller and McMahan, 1976).

Vesicle fields varied considerably in extent, even in two boutons belonging to a single arbor (Figure 3). Mitochondria lay nearby to nearly all vesicle fields (Fig. 3A) with the exception of some smaller vesicle fields (Figure 3B). Although most arbors contained presynaptic boutons interconnected by thinner $(<1 \mu \mathrm{m}$ in diameter) branches, occasionally thicker $(>2$ $\mu \mathrm{m}$ in diameter) processes had vesicle fields as well, often at branching sites (Figure 3C).

Most arbors contained synaptic vesicles that were densely packed within a bouton along with mitochondria and other structures (Figures 2, 3, 7). In some arbors, however, vesicle fields inhabited small regions relative to their containing process (Figure 4). In these arbors we frequently observed two or more vesicle fields in a single section (arrows, Figure 4).

Presynaptic boutons were linked together by thinner inter-bouton processes $(<1 \mu \mathrm{m}$ in diameter) (Figure 2E). In some instances we could trace back from a presynaptic bouton to the point where the small branch split off from a larger process of the neuron. These thicker processes ( $>2 \mu \mathrm{m}$ in diameter) were typically devoid of small synaptic vesicles (Figure $5 \mathrm{~A}$ ), though some did contain them (Figure 3C). Within the imaged volume, we also observed a single large branch that contained several small vesicle fields yet did not appear to be involved in any synaptic activity, given the lack of any apposed postsynaptic arbors in their vicinity (Figure 5B). In this case, the smaller branches of this cell formed presynaptic arrangements comprising several relatively small vesicle fields similar to those documented in Figure 4.

Large vesicles-As noted previously, some arbors contained a population of larger dense core vesicles. These larger vesicles were often found surrounding fields of small presynaptic vesicles, though we also found them in relative isolation, scattered throughout the containing arbor. We broadly divided the arbors containing these dense core vesicles into two categories on the basis of how intensely the vesicles stained.

We found some arbors that contained intensely stained dense core vesicles $100 \mathrm{~nm}$ in maximal diameter with cross-sectional shapes ranging from circular to ovoid (Figure 6). If the arbor contained presynaptic boutons, these vesicles were found in aggregations adjacent to-and partially overlapping with - the fields of small vesicles (Figure 6A, B). In general, presynaptic boutons of this arbor type were smaller and contained smaller presynaptic vesicle fields (see Figure 8). Aggregations of the larger intensely stained dense core vesicles were also occasionally encountered in the absence of any immediately adjacent small vesicle fields. In between presynaptic boutons, these arbors contained scattered dense core vesicles (Figure 6C, D). Within the whole image volume, we also found a single arbor that contained the intensely stained dense core vesicles scattered within large branches and throughout all of its thinner branches, even though the arbor lacked any boutons containing fields of small synaptic vesicles (Figure 6E). 
We also found arbors that contained a population of large (100 to $170 \mathrm{~nm}$ in maximal diameter), close-packed vesicles. Unlike the intensely staining dense core vesicles, the maximal diameters of these vesicles were larger and the vesicles were more lightly stained (Figure 7A-C). These large, low electron density vesicles were sometimes associated with presynaptic vesicles within large boutons over $2 \mu \mathrm{m}$ in diameter (Figure 7C). Aggregations of these large vesicles were also sometimes found independently of small vesicles and scattered in the thin processes connecting presynaptic boutons (arrowheads, Figure 7B). Occasionally, scattered glycogen particles are observed interspersed among these vesicles (Figure 7C), a pattern also observed by earlier researchers (Coggeshall and Fawcett, 1964).

We were interested in how frequently we observed arbors that contained either the intensely stained dense core vesicles or the large low density vesicles. We conducted an analysis where we visually inspected the arbor containing a randomly generated point in the volume and determined whether or not it contained vesicles. Of the vesicle-containing arbors, $70 \%$ (92/131) did not contain aggregations of either large low density vesicles or large dense core vesicles (though some of these did contain scattered larger low density vesicles as in Figure $2)$. Four percent (5/131) contained intensely stained dense core vesicles and the remaining $26 \%(34 / 131)$ contained aggregations of large low density vesicles of varying staining intensity (Figure 7A, C).

We next asked whether the vesicle content of a given bouton influenced the number of postsynaptic partners it had. For this analysis, we outlined the fields of the small presynaptic vesicles at 59 boutons each taken from one of the sets of arbors we analyzed in our random point analysis. In addition, we included more samples of selected boutons containing intensely stained dense core vesicles and large low density vesicles as there were relatively few of these in the randomly-sampled dataset. We then estimated the volume of the presynaptic vesicle fields by multiplying their cumulative imaged area by the slice thickness $(70 \mathrm{~nm})$. We next plotted the volume of the synaptic vesicle fields versus the number of postsynaptic contacts (Figure 8). Different sized and colored circles indicate whether the bouton contained primarily small synaptic vesicles (small grey dots), large intensely stained dense core vesicles in addition to small synaptic vesicles (black dots), or large low density vesicles in addition to small synaptic vesicles (large open circles). We found that boutons that contained intensely stained dark vesicles tended to have smaller synaptic vesicle fields and have subsequently have fewer postsynaptic partners (black dots, Figure 8).

\section{Juvenile Ganglion}

Vesicle-containing and vesicle-lacking arbors-After analyzing the volume of adult neuropil, we sectioned and imaged a volume spanning an entire ganglion. To reduce acquisition time, we selected a ganglion from the juvenile leech. Juvenile leeches perform most of the same behaviors studied in the adult (Reynolds et al., 1998) yet are much smaller and possess ganglia with diameters $60 \%$ of their adult counterparts. Previous work has shown that neuronal arbors in the juvenile leech exhibit the same shape and branching pattern as those same arbors in adults, though branch length and varicosity number increase with the increased size of adult arbors (DeRiemer and Macagno 1989). When we performed the same random point analysis in this volume as we did in the adult, we found that $78 \%$ 
(115/147) of neuronal arbors contained vesicles, while the remaining 22\% (32/147) did not. Compared to the adult volume, which contained arbors from within only a small region of the neuropil, this represented an additional $10 \%$ in the fraction of arbors without vesicles.

Number of somata and somal vesicle content-Because the juvenile material contained the entire ganglion, we were able to map every cell body. We found 397 neurons in total: 72 in the right anterolateral packet, 71 in the left anterolateral packet, 75 in the right posterolateral packet, 78 in the left posterolateral packet, 45 in the posteromedial packet, 49 in the anteromedial packet, and 7 neurons whose somata resided just inside the inner capsule and were thus technically in the neuropil (these neurons were first noted by Coggeshall and Fawcett [1964], but to our knowledge have since been ignored). The total number of neurons reported here is in line with previous estimates of 350 (Ort et al., 1974) and 400 (Macagno, 1980) in Hirudo medicinalis. Macagno (1980) counted neurons from several adult ganglia stained with osmium tetroxide and cut into thick serial sections. Using this approach he counted 400 neurons in ganglion 11, and a range of 389 to 398 neurons in ganglion 10. We imaged ganglion 11 from our juvenile animal.

Of these somata, we also found a few that contained either large intensely stained dense core vesicles or large low density vesicles (Figure 9) like the arbors described from the adult volume (Figures 6,7). Based on the locations of these neurons (Figure 9C, blue neurons), the neurons containing the intensely staining vesicles correspond to the six serotonergic neurons of midbody ganglia according to previous observations (Trueta et al., 2012; Lent et al., 1991). Many of the somata containing large low density vesicles do not match any previously-identified neurons (Figure 9C, red neurons), although two of these neurons are very likely the Leydig cells (Figure 9B, C), based on their size and soma position.

\section{Location of synaptic inputs and outputs within fully reconstructed arbors-}

Because we could map every neuronal soma in the ganglion (Figure 10A), we were able to identify neurons of interest, which could then be traced and annotated fully. To confirm a candidate neuron's identity, we first compared the location and size of its soma to existing maps (Ort et al., 1974). Cell body position is somewhat variable from ganglion to ganglion. However, adding neuronal arbor morphology enabled the positive identification of one neuron among its neighbors (Fan et al., 2005). Importantly, neuropilar arbor shape is established during embryogenesis, prior to the juvenile life stage (DeRiemer and Macagno, 1989; Marin-Burgin et al., 2006). We therefore based our cell identifications on soma size, location, and arbor morphology. To demonstrate this, we selected the inhibitory motor neuron DI-1 (dorsal inhibitor 1) because many of its physiological connections have been characterized (Ort et al., 1974; Kristan et al., 2005). We reconstructed the entire arbor of the right DI-1 in the juvenile ganglion (Figure 10B). We also generated a skeleton model of DI-1 by placing a single dot within the contour of a neuron's membrane in a given section of the volume (each dot, or node, is connected to the next via a line when represented graphically) (Figure 10C). Tracing arbors in this manner is common (e.g. White et al., 1986; Briggman et al., 2011; Ohyama et al., 2015) as it reduces segmentation time considerably. It should be noted however that while this skeletonizing process captures the neuron's entire arbor, it does generate some false branches in thick processes that travel roughly parallel to 
the imaging plane. While we could have removed these by removing the nodes of these short branches, in many cases doing so would risk leaving unlabeled a region of an arbor that contained pre- or postsynaptic zones, so we chose to leave the skeletons unedited. We then annotated this arbor by marking locations on the skeleton where we found presynaptic and postsynaptic sites, using the criteria described previously (Figure 2). On the skeleton of neuron DI-1, presynaptic sites were marked with red balls and postsynaptic sites with green balls (Figure 10C). For this neuron, outputs were lateralized to the contralateral half of the arbor, while inputs were found throughout.

The total number of input and output sites are summarized for the neurons we traced and fully annotated (Table 1). The total number of synaptic inputs to the right DI-1 shown is 912 (Table 1). Presynaptic boutons can form dyadic synapses onto multiple postsynaptic partners (Figure 2) and can vary greatly in size (Figure 3). The reconstructed cell DI-1 had 261 presynaptic boutons, each of which had multiple potential postsynaptic targets (a range of 1 to 21 , with a median and mode of 7) for a total of 1684 potential postsynaptic processes (Table 1).

Neuron DI-1 is an inhibitory motor neuron that inhibits dorsal longitudinal muscles in the body wall directly as well as inhibiting excitatory motor neurons within the central neuropil (Ort et al., 1974; Cline et al., 1985). To determine whether the locations of synaptic input and output within DI-1 (Figure 10C) are a pattern common to other motor neurons, we traced the inputs and outputs of other motor neurons, both inhibitors and excitors, of the same longitudinal muscles. Not surprisingly, we found that the DI-1 on the opposite (left) side, was a mirror image of the right DI-1 neuron, and that another inhibitor of the dorsal longitudinal muscles (the pair of DI-102 neurons) also had a zone ipsilateral to the soma with extensive synaptic input and a contralateral zone of mixed inputs and outputs (Figure 11A). A pair of excitatory motor neurons to the same dorsal longitudinal muscles (the DE-3 neurons) largely lacked presynaptic vesicles with a few exceptions on the contralateral half of their arbors (Figure 11B). These connectivity patterns are consistent with physiological observations: DE-3 makes no known chemical synaptic connections within the ganglion (Ort et al., 1974), and DI-1 inhibits excitatory motor neurons exclusively by connections on the contralateral side (Lytton and Kristan, 1989). We also examined motor neurons that innervate the ventral longitudinal muscles. Neurons VI-2 inhibit these muscles and excitatory motor neurons analogously to DI-1, and these neurons also contain an ipsilateral portion of their arbor that is entirely postsynaptic and a contralateral portion of mixed postsynaptic and presynaptic function. Neurons VE-4, a pair of excitatory motor neurons presynaptic to the ventral longitudinal muscles, have an exclusively ipsilateral arborization within the neuropil (Fan et al., 2005). We found that their arbors contained only postsynaptic connections.

Not all inhibitory neurons segregated their input and output arbors on the left and right sides as did DI-1, DI-102, and VI-2. Cells 116, a pair of neurons that inhibit both dorsal and ventral motor neurons (E.P. Frady and K. Todd, personal communication), arborize in both the ipsilateral and contralateral neuropil like these inhibitory motor neurons, but unlike them, neurons 116 had sites of synaptic input and output throughout both halves of their arbors (Figure 11C). Similarly the S cell, an interneuron involved in the shortening reflex of 
the leech known to make only excitatory connections (Frank et al., 1975; Magni and Pellegrini, 1978; Muller and Scott, 1981; Crisp and Muller, 2006), had both inputs and outputs throughout all the branches of its arbor (Figure 11D). These branches descend from the $\mathrm{S}$ cell's thick axon, which then travels outward in both directions from the ganglion in Faivre's nerve (Laverack 1969; Frank et al., 1975; Muller and Carbonetto, 1979).

\section{DISCUSSION}

Among the early investigators to use electron microscopy to investigate the leech nervous system, Coggeshall and Fawcett (1964) wrote of the neuropil: "The complexity of this region and the sampling problem inherent in electron microscopy make it impossible at present to provide a functionally meaningful account of the synaptic relationships within the neuropil, or even to identify the source of the various categories of nerve processes. Until means are devised for overcoming this sampling problem [...] the morphologist must be content to describe the cytological characteristics of the glial cells and the fine structure of the various nerve processes and synaptic complexes encountered here." In our work, we applied SBEM to solve this "sampling problem" and begin describing the features of neuronal arbors within the ganglion's neuropil. We sampled both a small region of adult neuropil and an entire juvenile ganglion (containing both the outer layer of cell bodies and the inner neuropilar zone). These are the first large serial EM datasets generated from leech and represent the first time entire ganglion-contained arbors of leech neurons have been reconstructed from data containing this level of detail.

Within both volumes, most arbors contained fields of synaptic vesicles, while a smaller fraction did not. In the juvenile ganglion, we could trace entire arbors, revealing that some completely lack any vesicles or presynaptic varicosities in the ganglion (the VE-4s), or almost entirely lacked vesicles (the DE-3s). Other neurons segregated their arbors, with the contralateral half full of vesicle-containing varicosities (DI-1s, VI-2s) while the ipsilateral half remained devoid of vesicles. Still others possessed vesicle-containing varicosities in every branch of their arbor (the inhibitory $116 \mathrm{~s}$, and the excitatory $\mathrm{S}$ cell). The various combinations of vesicle-containing and vesicle-lacking processes in the arbors we traced suggests that the leech neurons have the capability to spatially segregate their arbors into input-only zones and mixed input and output zones (notably, we found no example of an arbor or portion thereof that was only presynaptic to other cells). The ability to form output sites within only specific regions of neuronal arbors is a hallmark of nervous systems across phylogeny, including C. elegans (White et al., 1986), Drosophila (Takemura et al., 2013; Chen et al., 2013; Ohyama et al., 2015; Scheider-Mizell et al., 2016), and vertebrates, where axons and dendrites are well-established as distinct domains. Mixed input and output zones are also common. For instance, certain laminar and medullar neurons in Drosophila possess a limited number of synaptic outputs in what's otherwise characterized as a "dendritic" zone (Takemura et al., 2013; Chen et al., 2013). Similarly, many of the neurons fully reconstructed in the Drosophila larva also possess mixed input and output zones (Ohyama et al., 2015; Berck et al., 2016; Schneider-Mizell et al., 2016). In the locust, neurons have been described in which inputs and outputs are intermingled among the same branches (Watson and Burrows, 1982). In the crayfish, inputs onto and outputs from a single neuron were found in close proximity on the same branches (Kondoh and Hisada, 1986). In the lobster 
stomatogastric ganglion, all arbors have been found to contain both sites of synaptic input and output, with the exception of the gastric mill neurons, which lacked sites of synaptic output (King, 1976a, 1976b). In Aplysia single cells have been shown to make output and receive inputs on the same branches (Gillette and Pomeranz, 1975; Bailey et al., 1979). And in the Platynereis visual system, all synapses are considered axo-axonal (Randel et al., 2014).

Structurally, vesicle-lacking processes in our volumes branched prolifically with many small extensions near the tips of branches that received synaptic input from a contacting presynaptic varicosity belonging to another arbor. It was not uncommon to find processes of one of these arbors that contacted the same presynaptic bouton more than once (Figure 2). This echoes findings in other systems, including crustaceans (Sandeman and Mendum, 1971; King 1976a), mollusks (Bailey et al., 1979), insects (Watson and Burrows, 1982), and mammals (Wilke et al, 2013; Kasthuri et al., 2015).

Vesicle-containing arbors were diverse, containing small presynaptic vesicles that were aggregated into fields within boutons and varicosities. Earlier efforts in the leech distinguished the small presynaptic vesicles as being either granular or agranular (Muller and McMahan, 1976; Muller 1979) and work in the crustacean stomatogastric ganglion has similarly differentiated small vesicles on the basis of their size and shape (King 1976a; Kilman and Marder, 1996). While such fine differences were likely present in our samples, the resolution limits inherent to SBEM preclude us detecting them. We do observe some differences in vesicle-containing arbors that become apparent when these can be studied across many serial images. For instance, most of these arbors contain vesicles within boutons or varicosities that are linked by thinner processes $(<1 \mu \mathrm{m}$; Figure $2 \mathrm{E})$ and at these sites the presynaptic vesicles, larger vesicles, and mitochondria together densely fill the cytoplasm of the process (Figures 2,3). Among the arbors we traced in the juvenile ganglion, cells 116, DI-1, VI-2, DE-3, DI-102 all display this pattern in their secondary branches. This arrangement of intracellular components is typical of presynaptic structure in other species, including crustaceans, mollusks, and insects (Sandeman and Mendum, 1971; King, 1976a, 1976b; Kilman and Marder, 1996; Bailey et al., 1979; Meinertzhagen 1996; Watson and Burrows 1982, 1983, 1985).

In some vesicle-containing arbors in our volumes, vesicle fields and surrounding mitochondria did not fill their containing bouton, and often multiple smaller vesicle fields could be observed within the same section (Figure 4). In the juvenile ganglion, we observed this pattern in the $\mathrm{S}$ cell and the two interneurons known as the coupling interneurons because of how strongly electrically-coupled they are to the S cell (Muller and Scott, 1981). While this pattern has not been assigned to specific cells in previous work, some examples are found in images of crustacean neuropil (Figure 4 in Sandeman and Mendum, 1971; Figure 7 in King 1976a; Figure 2 in Kilman and Marder, 1996), and in the locust (Figures 4 and 5 in Watson and Burrows, 1982). It is unclear what functional ramifications these unfilled boutons present.

While many arbors contained a population of scattered larger (100nm maximal diameter) vesicles surrounding their pools of small presynaptic vesicles, we found a few arbors that 
contained substantial aggregations of larger vesicles. With our staining protocol, there appear to be at least two basic types of these vesicles: intensely stained (maximal diameter $100 \mathrm{~nm}$ ) and lightly stained (maximal diameter $170 \mathrm{~nm}$ ). Some of the intensely stained dense core vesicles likely contain serotonin on the basis of the pattern of somata containing them in our juvenile ganglion volume (Lent et al., 1991) in accordance with prior evidence (Muller, 1979; Kuffler et al., 1987; Trueta et al., 2012). Intensely stained dense core vesicles are a hallmark of serotonergic vesicles, as described in the crustacean stomatogastric ganglion (King, 1976a, 1976b; Friend, 1976) and mollusk (Cottrell and Osborne, 1970; Goldman et al., 1976). Dense core vesicles are also known to contain other monoamines, including the neurotransmitters dopamine (Séguéla et al., 1988) and octopamine (Lee and Wyse, 1991) known to be present in leech neurons (Crisp et al., 2002). The neurons that stain for markers of these neurotransmitters do not have cell bodies in ganglion 11 (Crisp et al., 2002), which is consistent with our observation that intensely stained dense core vesicles were observed only in the somata of serotonergic neurons. However, dopaminergic and octopaminergic neurons do send processes from peripheral ganglia and from the head and tail brains into the segmental ganglia. Therefore some of the intensely stained dense core vesicles that belong to processes we observe entering the ganglion via the nerve roots or connectives could contain these neurotransmitters and should not be considered exclusively serotonergic.

Both the intensely stained dense core vesicles and low density large vesicles were found in four arrangements: in aggregations surrounding fields of smaller vesicles, in aggregations by themselves within the arbor, in aggregations by themselves in the soma, or in isolation within the arbor. Similarly, dense core vesicles are present in the crustacean stomatogastric ganglion in somata, scattered in neuronal processes, and in large aggregations in neuronal processes (Friend, 1976; Kilman and Marder, 1996). Aggregations of dense-cored vesicles are common to many nervous systems, observed also in C. elegans (White et al., 1986), Aplysia (Coggeshall, 1967; Gillette and Pomeranz, 1975; Bailey et al., 1979), the snail Helix pomatia (Elekes and S.-Rózsa 1984), and the locust (Watson and Burrows, 1985). It is unclear which of these arrangements, if any, are most likely to be sites of exocytosis. While sites of large aggregations in the neuropil are likely candidates (e.g. Kilman and Marder, 1996), in the leech previous work has shown that, in the Retzius cells, serotonin from large dense core vesicles can also be released from the somata themselves (Bruns and Jahn, 1995; Trueta et al., 2012). These observations underscore a major challenge in using reconstructions of neurons to precisely model their influence on other cells: it is difficult to determine where exactly the contents of neurosecretory vesicles are released or which of the myriad arbors nearby are influenced by the diffusion of their contents. In $C$. elegans and the crustacean stomatogastric ganglion, systems with known connectivity maps, neuromodulators released by neurosecretory vesicles have been shown to exert long-range influence and reconfigure the activity patterns of neural circuits (Leinwand and Chalasani, 2012; Bargmann and Marder, 2013). Eventually, analysis of serial EM datasets must also estimate the particular shape of extracellular spaces present (as in Kinney et al., 2013) though accurately achieving this goal requires advances in fixation techniques (Korogod et al., 2015; Pallotto et al., 2015). 
We classified chemical synapses on the basis of vesicle proximity to membrane appositions using some existing conventions in the leech (Muller and McMahan, 1976; Muller 1979) and subsequently studied the 3-dimensional features of the presynaptic varicosities and postsynaptic arbors involved (Figure 2). The typical leech presynaptic bouton lies in apposition to many postsynaptic processes (median of 7 in a random sample of presynaptic boutons within the adult neuropil, Figure 8). The number of postsynaptic partners per bouton reported here is generally higher than what has been previously reported. For instance, in Aplysia sensory neurons were found to synapse onto either one or two postsynaptic targets (Bailey et al., 1979). Similarly, in C. elegans the typical synaptic arrangement involves one or at most three postsynaptic partners (White et al., 1986). In the crustacean stomatogastric ganglion, synaptic arrangements are reported to typically contain two or three postsynaptic partners (King, 1976a; Kilman and Marder, 1996), though King (1976a) did report a single presynaptic bouton with 18 postsynaptic contact points after reconstructing it from serial sections. In the locust, postsynaptic processes are observed commonly in pairs (Watson and Burrows, 1982, 1983, 1985). Synapses in the visual ganglion of the housefly have been reported to involve four postsynaptic processes per presynaptic element (Burkhardt and Braitenberg, 1976). Similarly, in dense serial TEM reconstruction of Drosophila, Takemura and co-workers (2013) report 3-5 postsynaptic processes per presynaptic bouton. Recent connectomic efforts in the Drosophila larva also indicate multiple postsynaptic partners at each bouton in both the brain (Berck et al., 2016) and the abdominal segments (Ohyama et al., 2015; Schneider-Mizell et al., 2016). These differences in postsynaptic process number per synapse may be related to the more liberal classification scheme used in this work, which relies on proximity of vesicles and membrane apposition as the direct observation of presynaptic structures like tufts or bars is impossible at SBEM resolution in leech tissue.

The ability to trace full arbors and locate the synaptic connections among them is one of the great promises of SBEM and ssTEM and is essential for connectomics (e.g. White et al., 1986; Bock et al., 2011; Briggman et al., 2011; Helmstaedter et al., 2013; Randel et al., 2014; Ohyama et al., 2015; Berck et al., 2016). However, the dividends of SBEM and ssTEM extend beyond analysis of connectivity. The potential to reconstruct the full structure of neurons and their intracellular components also offers rich rewards. Reconstructions from serial EM datasets can be used to predict the diffusion of molecules in the extracellular spaces of neuropil (Kinney et al., 2013), the diversity of presynaptic structures (Wilke et al., 2013), or locate proteins of interest tagged with markers that differentiate them in EM (Shu et al., 2011; Atasoy et al., 2014; Lam et al., 2015). Here we focused on describing the diversity of vesicle arrangements and localization of presynaptic boutons and synaptic inputs found within arbors of leech neurons. This information can in turn be correlated with neurotransmitter contents (in the case of the serotonergic intensely staining dense core vesicles) or reveal which portions of an arbor receive only synaptic inputs and which also generate outputs. These factors are essential for spatially detailed modeling of a given neuron's electrical behavior. The location of synaptic inputs relative to the production of synaptic outputs determines in part how strongly presynaptic neurons influence their postsynaptic partners. Retaining this three-dimensional information therefore can only strengthen what would otherwise be reduced to one dimension in a connectome, and is essential to understanding how neural circuits produce behavior. 


\section{Acknowledgments}

Grant sponsor: National Institutes of Health; Grant number: MH43396 (to WBK);

Grant sponsor: National Institutes of Health; Grant number: P41GM103412 (to MHE)

Grant sponsor: National Science Foundation; Grant number: IOS 825741 (to WBK);

Grant sponsor: Kavli Institute for Brain and Mind (to WBK and MHE)

We thank Mason Mackey and Tom Deerinck for their assistance and advice for preparing and imaging the samples. This work was supported by an NIH research grant (MH43396) to WBK., an NSF grant (IOS 825741) to WBK, an NIH grant (P41GM103412) to MHE, and a grant from the Kavli Institute for Brain and Mind (to WBK and MHE).

\section{LITERATURE CITED}

Atasoy D, Betley JN, Li W-P, Su HH, Sertel SM, Scheffer LK, Simpson JH, Fetter RD, Sternson SM. A genetically specified connectomics approach applied to long-range feeding regulatory circuits. Nat Neuro. 2014; 17(12):1830-1839.

Bailey CH, Thompson EB, Castellucci VF, Kandel ER. Ultrastructure of the synapses of sensory neurons that mediate the gill-withdrawal reflex in Aplysia. J Neurocyt. 1979; 8(4):415-444.

Bargmann CI, Marder E. From the connectome to brain function. Nat Meth. 2013; 10(6):483-490.

Berck ME, Khandelwal A, Claus L, Hernandez-nu L, Si G, Tabone CJ, Li F, Truman JW, Fetter RD, Louis M, Samuel ADT, Cardona A. The wiring diagram of a glomerular olfactory system. eLife. 2016; 2016(5):e14859.

Bock DD, Lee W-CA, Kerlin AM, Andermann ML, Hood G, Wetzel AW, Yurgenson S, Soucy ER, Kim HS, Reid RC. Network anatomy and in vivo physiology of visual cortical neurons. Nature. 2011; 471(7337):177-182. [PubMed: 21390124]

Briggman KL, Bock DD. Volume electron microscopy for neuronal circuit reconstruction. Curr Opinion Neurobio. 2012; 22(1):154-161.

Briggman KL, Helmstaedter M, Denk W. Wiring specificity in the direction-selectivity circuit of the retina. Nature. 2011; 471(7337):183-188. [PubMed: 21390125]

Bruns D, Jahn J. Real-time measurement of transmitter release from single synaptic vesicles. Nature. 1995; 377(7):62-65. [PubMed: 7659162]

Burkhardt W, Braitenberg V. Some peculiar synaptic complexes in the first visual ganglion of the fly, Musca domestica. Cell and Tissue Res. 1976; 173:287-308. [PubMed: 991241]

Cardona A, Saalfeld S, Schindelin J, Arganda-Carreras I, Preibisch S, Longair M, Tomancak P, Hartenstein V, Douglas RJ. TrakEM2 software for neural circuit reconstruction. PLoS ONE. 2012; 7(6)

Chen Y, Akin O, Nern A, Tsui CYK, Pecot MY, Zipursky SL. Cell-type-specific labeling of synapses in vivo through synaptic tagging with recombination. Neuron. 2014; 81(2):280-293. [PubMed: 24462095]

Cline HT, Nusbaum MP, Kristan WB. Identified GABAergic inhibitory motor neurons in the leech central nervous system take up GABA. Brain Res. 1985; 348(2):359-362. [PubMed: 4075094]

Coggeshall RE. A light and electron microscope study of the abdominal ganglion of Aplysia californica. J Neurophysiol. 1967; 30(6):1263-1287. [PubMed: 6066444]

Coggeshall RE, Fawcett DW. The fine structure of the central nervous system of the leech Hirduo medicinalis. J Neurophysiol. 1964; 27(2):229-289. [PubMed: 14129772]

Cottrell GA, Osborne NN. Subcellular localization of serotonin in an identified serotonin-containing neurone. Nature. 1970; 225:470-472. [PubMed: 5411126]

Crisp KM, Klukas KA, Gilchrist LS, Nartey AJ, Mesce KA. Distribution and development of dopamine- and octopamine-synthesizing neurons in the medicinal leech. J Comp Neurol. 2002; 442(2):115-129. [PubMed: 11754166]

Crisp KM, Muller KJ. A 3-synapse positive feedback loop regulates the excitability of an interneuron critical for sensitization in the leech. J Neurosci. 2006; 26(13):3254-3531. 
Denk W, Horstmann H. Serial block-face scanning electron microscopy to reconstruct threedimensional tissue nanostructure. PLoS Bio. 2004; 2(11):e329-e329. [PubMed: 15514700]

DeRiemer SA, Macagno ER. Light microscopic analysis of contacts between pairs of identified leech neurons with combined use of horseradish peroxidase and Lucifer Yellow. J Neurosci. 1981; 1(6): 650-657. [PubMed: 7346573]

DeRiemer, SA.; Macagno, ER. Perspectives in Neural Systems and Behavior. New York: Alan R. Liss; 1989. Quantitative studies of the growth of neuronal arbors; p. 11-31.

Elekes K, Rosza S-K. Synaptic organization of a multifunctional interneuron in the central nervous system of Helix pomatia L. Cell and Tissue Research. 1984; 236:677-683. [PubMed: 6467342]

Fan R-J, Marin-Burgin A, French KA, Otto Friesen W. A dye mixture (Neurobiotin and Alexa 488) reveals extensive dye-coupling among neurons in leeches; physiology confirms the connections. $\mathrm{J}$ Comp Physiol A Neuroethol Sens Neural Behav Physiol. 2005; 191:1157-1171. [PubMed: 16133497]

Frank E, Jansen JKS, Rinvik E. A multisomatic axon in the central nervous system of the leech. J Comp Neurol. 1975; 159:1-13. [PubMed: 162801]

Friend BJ. Morphology and location of dense-core vesicles in the stomatogastric ganglion of the lobster, Panulirus interruptus. Cell and Tissue Res. 1976; 175(3):369-390. [PubMed: 187346]

Gillette R, Pomeranz B. Ultrastructural correlates of interneuronal function in the abdominal ganglion of Aplysia californica. J Neurobiol. 1975; 6(5):463-474. [PubMed: 1176980]

Goldman JE, Kim KS, Schwartz JH. Axonal transport of serotonin in an identified neuron of Aplysia californica. The journal of cell biology. 1976; 70:304-318. [PubMed: 1084888]

Granzow B, Friesen WO, Kristan WB Jr. Physiological and morphological analysis of synaptic transmission between leech motor neurons. J Neurosci. 1985; 5(8):2035-2050. [PubMed: 2991480]

Gray EG, Guillery RW. An electron microscopical study of the ventral nerve cord of the leech. Zeitschrift fur Zellforschung. 1963; 60:826-849.

Helmstaedter M, Briggman KL, Turaga SC, Jain V, Seung HS, Denk W. Connectomic reconstruction of the inner plexiform layer in the mouse retina. Nature. 2013; 500:168-174. [PubMed: 23925239]

French KA, Muller KJ. Regeneration of a distinctive set of axosomatic contacts in the leech central nervous system. J Neurosci. 1986; 6(2):318-324. [PubMed: 3950699]

Jarrell TA, Wang Y, Bloniarz AE, Brittin CA, Xu M, Thomson JN, Albertson DG, Hall DH, Emmons SW. The connectome of a decision-making neural network. Science. 2012; 337:437-444. [PubMed: 22837521]

Kilman VL, Marder E. Ultrastructure of the stomatogastric ganglion neuropil of the crab, Cancer borealis. J Comp Neurol. 1996; 374(3):362-375. [PubMed: 8906505]

King DG. Organization of crustacean neuropil. I. Organization of crustacean neuropil. I. Patterns of synaptic connections in lobster stomatogastric ganglion. J Neurocyt. 1976a; 5(2):207-237.

King DG. Organization of crustacean neuropil. II. Distribution of synaptic contacts on identified motor neurons in lobster stomatogastric ganglion. J Neurocyt. 1976b; 5(2):239-266.

Kinney JP, Spacek J, Bartol TM, Bajaj CL, Harris KM, Sejnowski TJ. Extracellular sheets and tunnels modulate glutamate diffusion in hippocampal neuropil. J Comp Neurol. 2013; 521(2):448-464. [PubMed: 22740128]

Kondoh Y, Hisada M. Distribution and ultrastructure of synapses on a premotor local nonspiking interneuron of the crayfish. J Comp Neurol. 1986; 254(2):259-270. [PubMed: 3794006]

Korogod N, Petersen CCH, Knott GW. Ultrastructural analysis of adult mouse neocortex comparing aldehyde perfusion with cryo fixation. eLife. 2015; 2015(4):e05793.

Kremer JR, Mastronarde DN, McIntosh JR. Computer visualization of three-dimensional image data using IMOD. J Struct Bio. 1996; 116(1):71-76. [PubMed: 8742726]

Kristan WB, Calabrese RL, Friesen WO. Neuronal control of leech behavior. Prog Neurobio. 2005; 76(5):279-327.

Lam SS, Martell JD, Kamer KJ, Deerinck TJ, Ellisman MH, Mootha VK, Ting AY. Directed evolution of APEX2 for electron microscopy and proximity labeling. Nature Methods. 2014; 12(1):51-54. [PubMed: 25419960] 
Laverack MS. Mechanoreceptors, photoreceptors and rapid conduction pathways in the leech, Hirudo medicinalis. J Exp Bio. 1969; 50(1):129-140. [PubMed: 5776582]

Leinwand SG, Chalasani SH. Neuropeptide signaling remodels chemosensory circuit composition in Caenorhabditis elegans. Nat Neuro. 2013; 16(10):1461-1467.

Lee HM, Wyse GA. Immunocytochemical localization of octopamine in the central nervous system of Limulus polyphemus: A light and electron microscopic study. J Comp Neurol. 1991; 207(4):683694.

Lytton WW, Kristan WB. Localization of a leech inhibitory synapse by photo-ablation of individual dendrites. Brain Res. 1989; 504(1):43-48. [PubMed: 2598016]

Macagno ER. Number and distribution of neurons in leech segmental ganglia. J Comp Neurol. 1980; 190(2):283-302. [PubMed: 7381060]

Macagno ER, Muller KJ, Pitman RM. Conduction block silences parts of a chemical synapse in the leech central nervous system. J Physiol. 1987; 387:649-664. [PubMed: 2821242]

Magni F, Pellegrino M. Patterns of activity and the effects of activation of the fast conducting system on the behaviour of unrestrained leeches. J Exp Biol. 1978; 76:123-135. [PubMed: 712325]

Marin-Burgin A, Eisenhart FJ, Kristan WB Jr, French KA. Embryonic electrical connections appear to pre-figure a behavioral circuit in the leech CNS. J Comp Physiol A Neuroethol Sens Neural Behav Physiol. 2006; 192(2):123-133. [PubMed: 16205960]

Meinertzhagen IA. Ultrastructure and quantification of synapses in the insect nervous system. $\mathbf{J}$ Neurosci Methods. 1996; 69(1):59-73. [PubMed: 8912936]

Meinertzhagen IA, O'Neil SD. Synaptic organization of columnar elements in the lamina of the wild type in Drosophila melanogaster. J Comp Neurol. 1991; 305(2):232-263. [PubMed: 1902848]

Muller KJ, Scott SA. Transmission at a direct electrical connexion mediated by an interneurone in the leech. J Physiol. 1981; 311:565-583. [PubMed: 6267257]

Muller KJ. Synapses between neurones in the central nervous system of the leech. Biol Rev Cambridge Phil Soc. 1979; 54(2):99-134.

Muller KJ, Carbonetto $\mathrm{S}$. The morphological and physiological properties of a regenerating synapse in the C.N.S. of the leech. J Comp Neurol. 1979; 185(3):485-516. [PubMed: 438368]

Muller KJ, McMahan UJ. The shapes of sensory and motor neurones and the distribution of their synapses in ganglia of the leech: a study using intracellular injection of horseradish peroxidase. Proc Royal Soc Lond B Biol Sci. 1976; 194(1117):481-499.

Ohyama T, Schneider-Mizell CM, Fetter RD, Aleman JV, Franconville R, Rivera-Alba M, Mensh BD, Branson KM, Simpson JH, Truman JW, Cardona A, Zlatic M. A multilevel multimodal circuit enhances action selection in Drosophila. Nature. 2015; 520:633-639. [PubMed: 25896325]

Ort CA, Kristan WB, Stent GS. Neuronal control of swimming in the medicinal leech II. Identification and connections of motor neurons. J Comp Physiol. 1974; 94:121-154.

Purves D, McMahan UJ. The distribution of synapses on a physiologically identified motor neuron in the central nervous system of the leech. J Cell Bio. 1972; 55(1):205-220. [PubMed: 4569409]

Randel N, Asadulina A, Bezares-Calderón LA, Verasztó C, Williams EA, Conzelmann M, Shahidi R, Jékely G. Neuronal connectome of a sensory-motor circuit for visual navigation. eLife. 2014; 2014(3):e02730.

Reynolds S, French K, Baader A, Kristan WB. Staging of middle and late embryonic development in the medicinal leech, Hirudo medicinalis. J Comp Neurol. 1998; 402(2):155-167. [PubMed: 9845240]

Sandeman DC, Mendum CM. The fine structure of the central synaptic contacts on an identified crustacean motoneuron. Zeitschrift für Zellforschung und Mikroskopische Anatomie. 1971; 119(4):515-525. [PubMed: 4327993]

Schneider-Mizell C, Gerhard S, Longair M, Kazimiers T, Li F, Zwart MF, Champion A, Midgley FM, Fetter RD, Saalfeld S, Cardona A. Quantitative neuroanatomy for connectomics in Drosophila. eLife. 2016; 2016:5, e12059.

Séguéla P, Watkins KC, Descarries L. Ultrastructural features of dopamine axon terminals in the anteromedial and the suprarhinal cortex of adult rat. Brain Res. 1988; 442(1):11-22. [PubMed: 3359247] 
Shu X, Lev-Ram V, Deerinck TJ, Qi Y, Ramko EB, Davidson MW, Jin Y, Ellisman MH, Tsien RY. A genetically encoded tag for correlated light and electron microscopy of intact cells, tissues, and organisms. PLoS Biology. 2011; 9(4)

Takemura, S-y; Bharioke, A.; Lu, Z.; Nern, A.; Vitaladevuni, S.; Rivlin, PK.; Katz, WT.; Olbris, DJ.; Plaza, SM.; Winston, P.; Zhao, T.; Horne, JA.; Fetter, RD.; Takemura, S.; Blazek, K.; Chang, L-A.; Ogundeyi, O.; Saunders, MA.; Shapiro, V.; Sigmund, C.; Rubin, GM.; Scheffer, LK.; Meinertzhagen, IA.; Chklovskii, DB. A visual motion detection circuit suggested by Drosophila connectomics. Nature. 2013; 500:175-181. [PubMed: 23925240]

Trueta C, Kuffler DP, De-Miguel FF. Cycling of dense core vesicles involved in somatic exocytosis of serotonin by leech neurons. Frontiers in Physiology. 2012; 3:1-13. [PubMed: 22275902]

Trujillo-Cenóz O. Some aspects of the structural organization of the intermediate retina of dipterans. J Ultrastructure Res. 1965; 13(1-2):1-33.

Wadepuhl M, Schäffner KH, Eberle R. An unusual cell in the central nervous system of Hirudo medicinalis L: a neuron with ribbons and flags. Cell and Tissue Res. 1990; 259(2):247-254.

Watson AH, Burrows M. The ultrastructure of identified locust motor neurones and their synaptic relationships. J Comp Neurol. 1982; 205(4):383-397. [PubMed: 7096627]

Watson AH, Burrows M. The morphology, ultrastructure, and distribution of synapses on an intersegmental interneurone of the locust. J Comp Neurol. 1983; 214(2):154-169. [PubMed: 6302137]

Watson AH, Burrows M. The distribution of synapses on the two fields of neurites of spiking local interneurones in the locust. J Comp Neurol. 1985; 240(3):219-232. [PubMed: 2415556]

White JG, Southgate E, Thomson JN, Brenner S. The structure of the nervous system of the nematode Caenorhabditis elegans. Phil Trans Royal Soc Lond B Biol Sci. 1986; 314:1-340.

Wilke SA, Antonios JK, Bushong EA, Badkoobehi A, Malek E, Hwang M, Terada M, Ellisman MH, Ghosh A. Deconstructing complexity: serial block-face electron microscopic analysis of the hippocampal mossy fiber synapse. J Neurosci. 2013; 33(2):507-522. [PubMed: 23303931] 

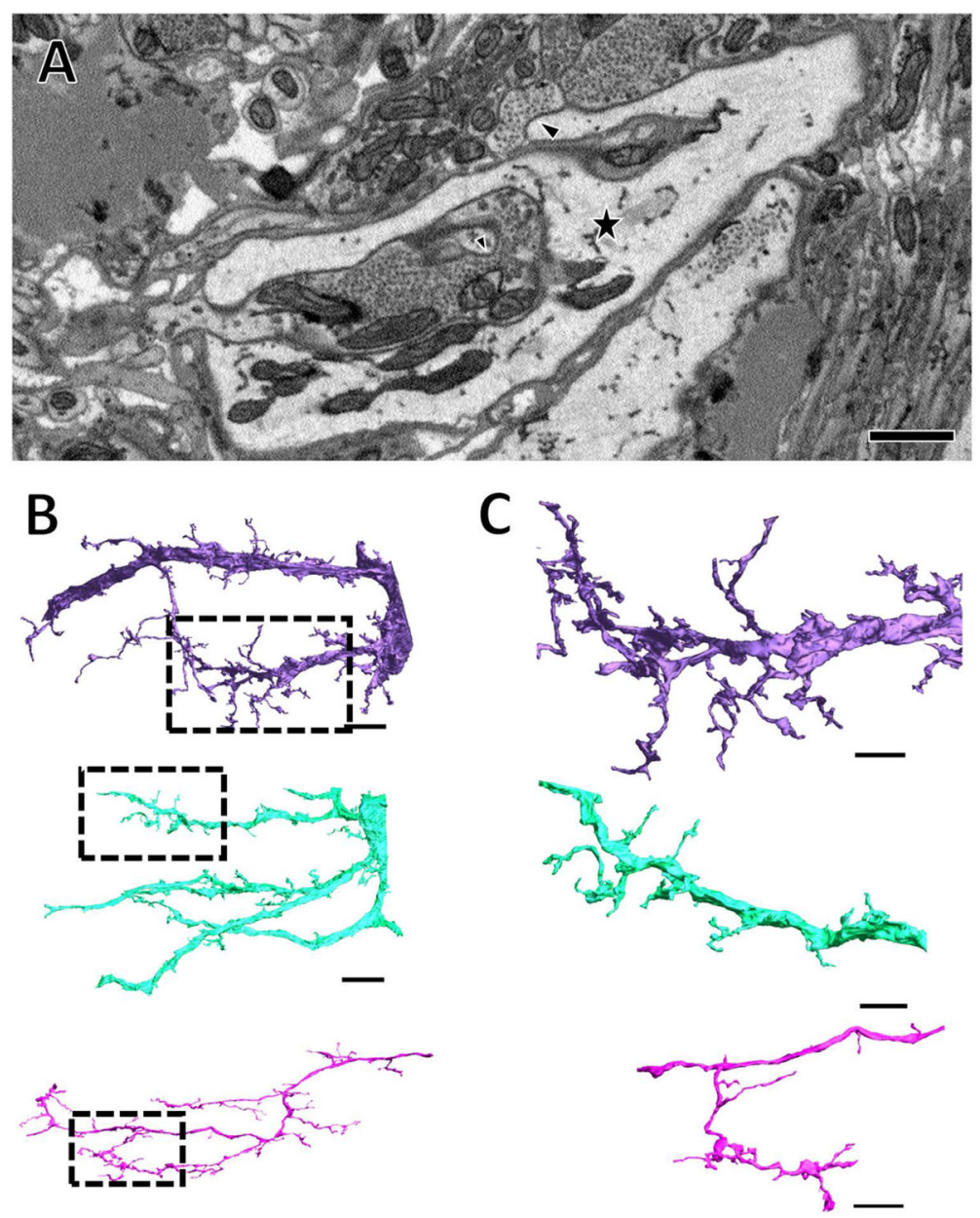

Figure 1.

Vesicle-lacking arbors branch profusely and make contact with many presynaptic boutons. (A) A single section of a vesicle-lacking arbor, denoted by the star symbol. A branch can be seen extending from the top of this process where it terminates opposite a presynaptic bouton (large arrowhead). In the center of the image, a small process that branched from the large starred process in another section terminates opposite another presynaptic bouton (small arrowhead). (B) Three vesicle-lacking arbors are shown reconstructed in 3D. The starred process shown in A belongs to the purple arbor here. (C) Closer views of the boxed regions in (B) reveal the fine structure of small processes. Scale bars $1 \mu \mathrm{m}$ in (A), $10 \mu \mathrm{m}$ in (B) and $5 \mu \mathrm{m}$ in (C). 

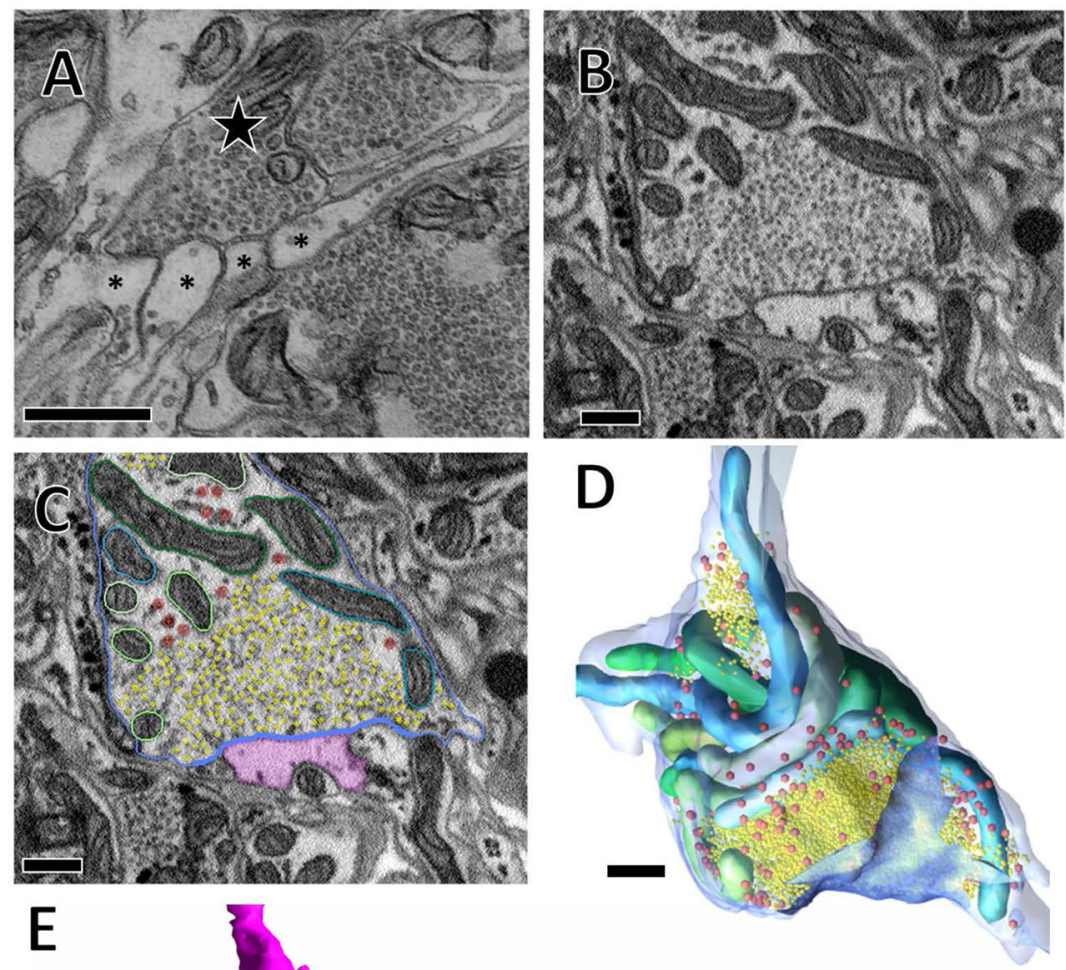

$\mathrm{E}$

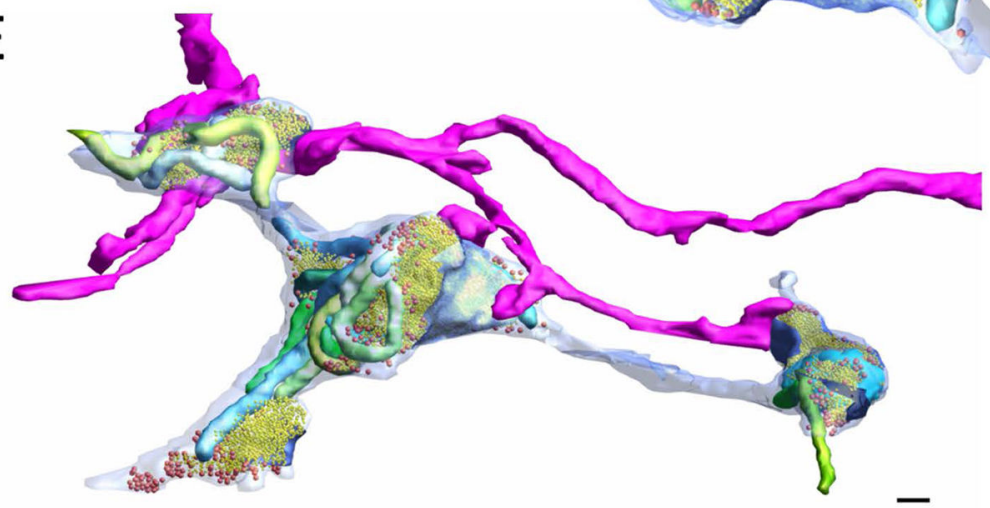

Figure 2.

In TEM and SBEM typical synaptic arrangements involve a vesicle-filled bouton in apposition to several smaller postsynaptic processes. (A) A thin section imaged with TEM showing the indenting apposition of numerous postsynaptic processes (asterisks) to a single presynaptic process (star) with some docked vesicles visible at these appositions. (B) A similar arrangement observed with SBEM. (C) The same image in (B) segmented so that the plasma membrane is blue; the region of plasma membrane in close proximity to synaptic vesicles is darker blue; synaptic vesicles are yellow; scattered larger vesicles are red; mitochondria are individually segmented in various shades of blue and green; and a postsynaptic process (same as in Figure 1B, C) is shown in pink. (D, E) Three-dimensional reconstructions of the bouton and others from the same cell along with the pink postsynaptic process that makes contact with several of these boutons. $500 \mathrm{~nm}$ scale bars in all panels. 

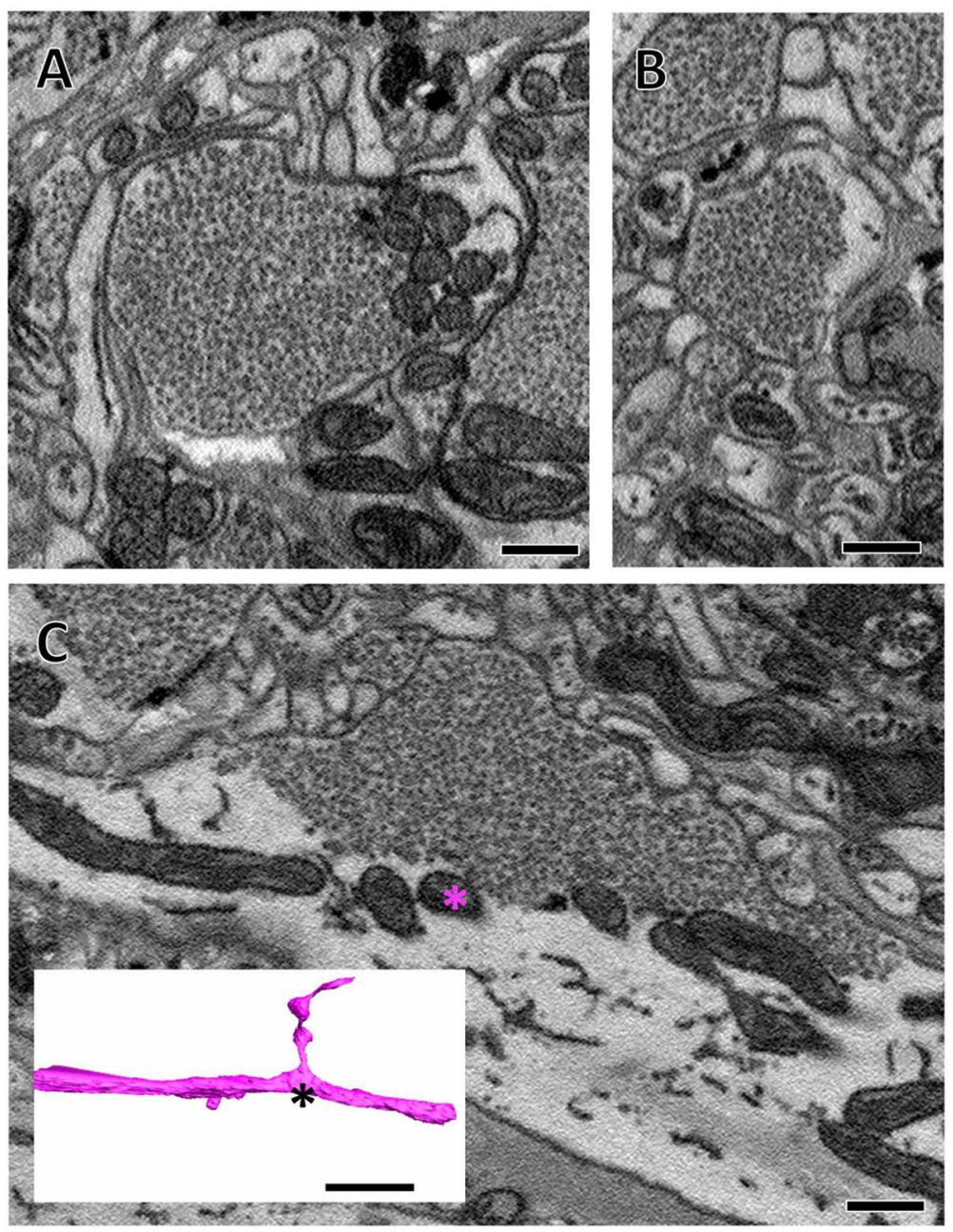

Figure 3.

Vesicle field extent varied within single arbors. (A-C) Individual boutons of a single arbor containing varying sizes of vesicle fields. In (C), the vesicle field is located at a branching site in the overall arbor, depicted in the inset. Scale bars $500 \mathrm{~nm}(\mathrm{~A}-\mathrm{C})$, inset in $(\mathrm{C})$ has 10 $\mu \mathrm{m}$ scale bar. 


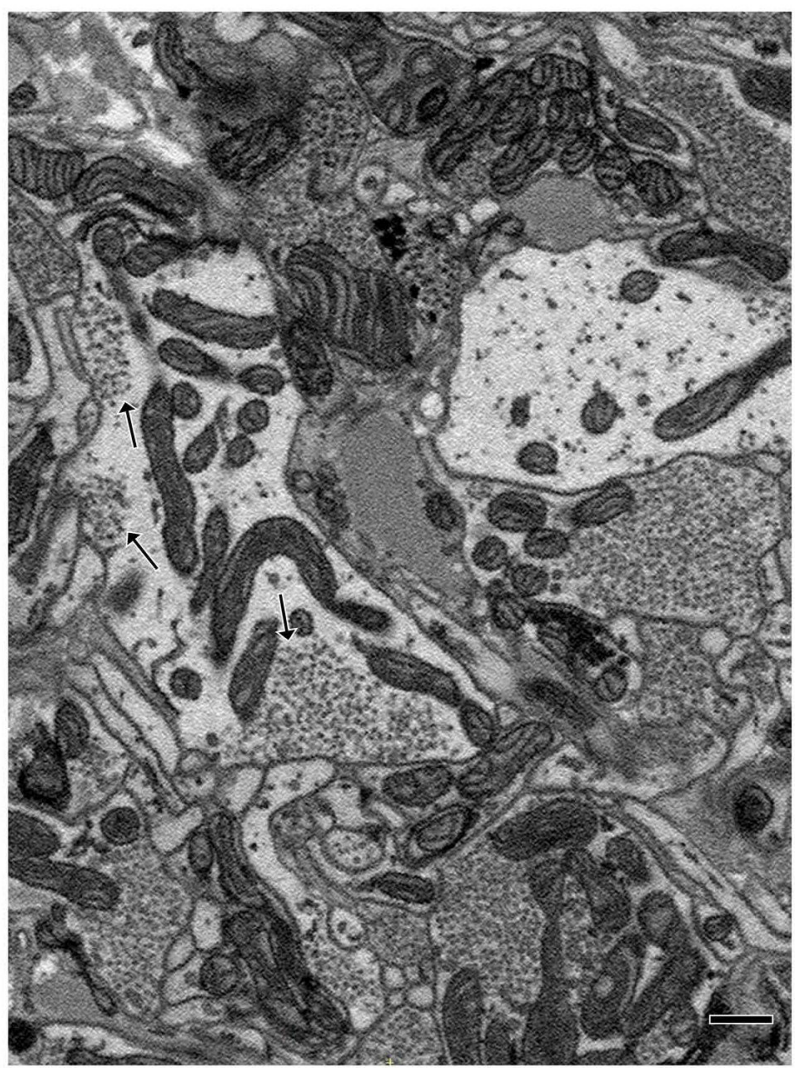

Figure 4.

Example of an arbor with vesicle fields that did not fill the entire process within which they were contained. As was common in such structures, this arbor had multiple vesicle fields visible in the same section (arrows). Scale bar $500 \mathrm{~nm}$. 

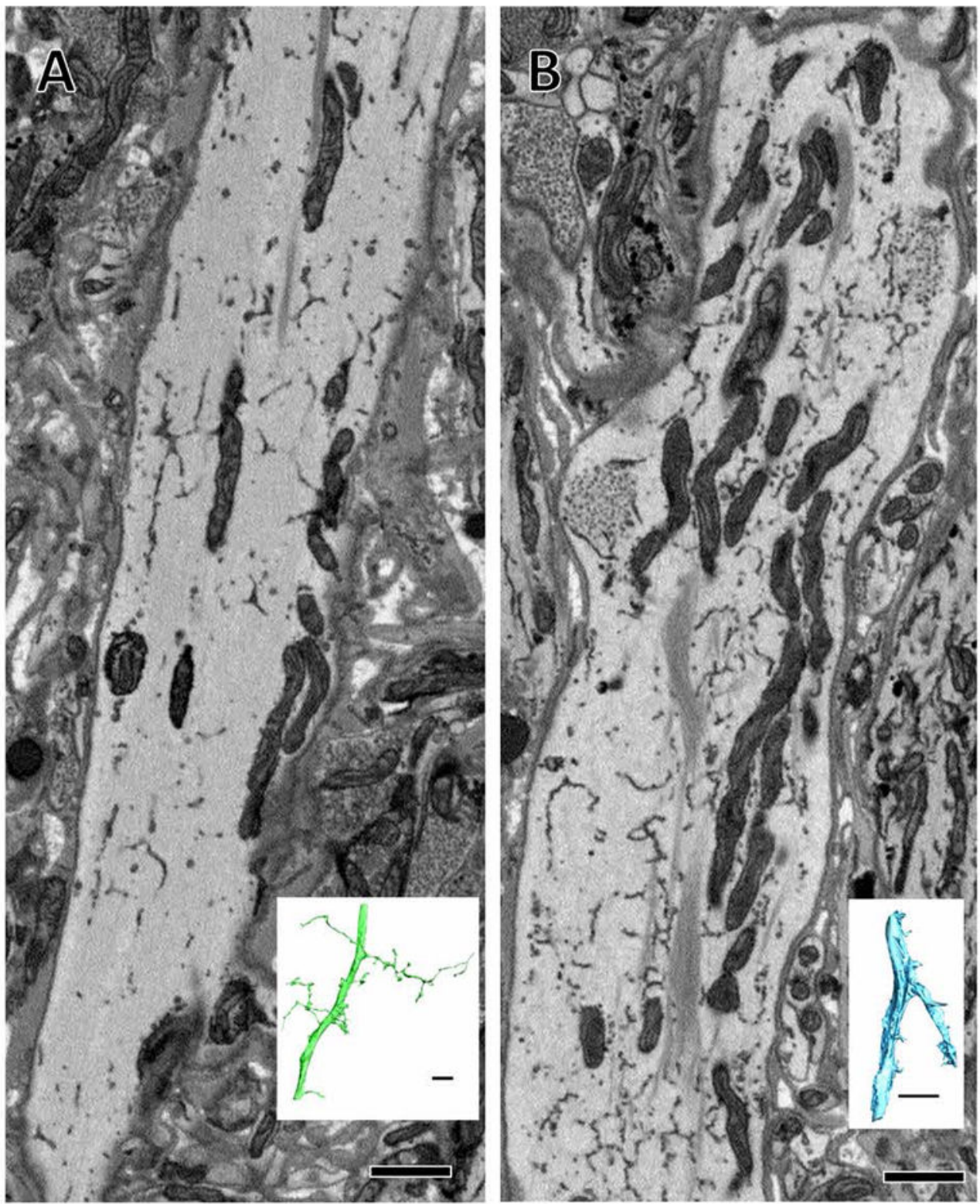

Figure 5.

Large processes belonging to vesicle-containing arbors in some cases contain vesicles. (A) A cross section of a large process belonging to a vesicle-containing arbor shown fully reconstructed in green in inset. No vesicles are present in this large process, though they are present in the arbor's smaller branches. (B) Another large process belonging to a vesiclecontaining arbor in which clusters of vesicles are visible (asterisks) yet are not presynaptic to any other arbor. Inset shows the full reconstruction of this arbor. Scale bars $1 \mu \mathrm{m}$ for micrographs, $10 \mu \mathrm{m}$ in insets. 

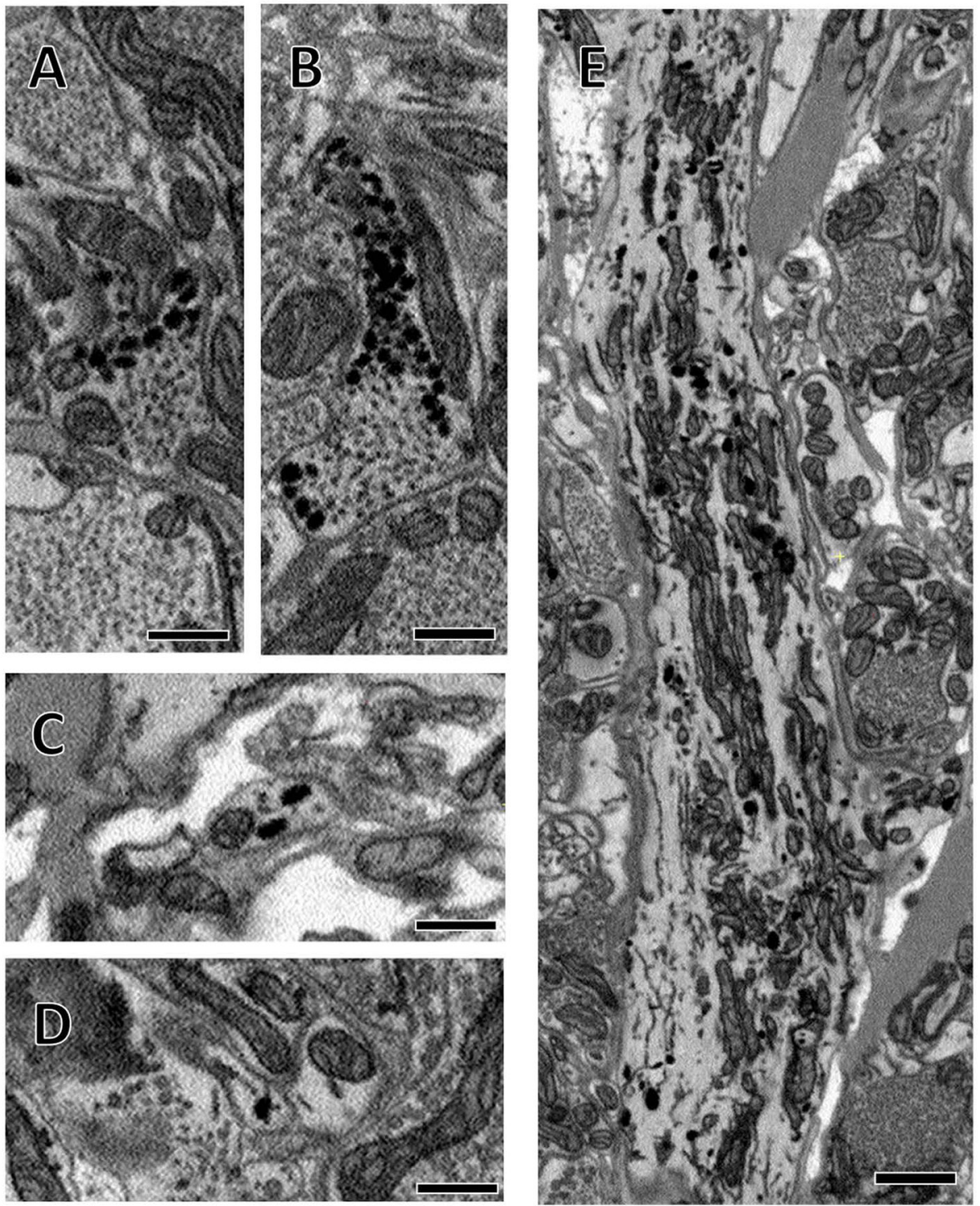

Figure 6.

Several examples of intensely staining dense core vesicles in various arbors. (A, B) Two boutons in which aggregations of these large dense core vesicles surround fields of presynaptic vesicles. (C, D) These large vesicles were found scattered throughout the thin processes that interlinked presynaptic boutons. (E) A large process of one arbor that contains many scattered large dense core vesicles. This particular arbor never formed any presynaptic boutons in the volume, though its secondary branches did contain scattered large dense core vesicles. (A-D) scale bars $500 \mathrm{~nm}$, (E) scale bar $1 \mu \mathrm{m}$. 

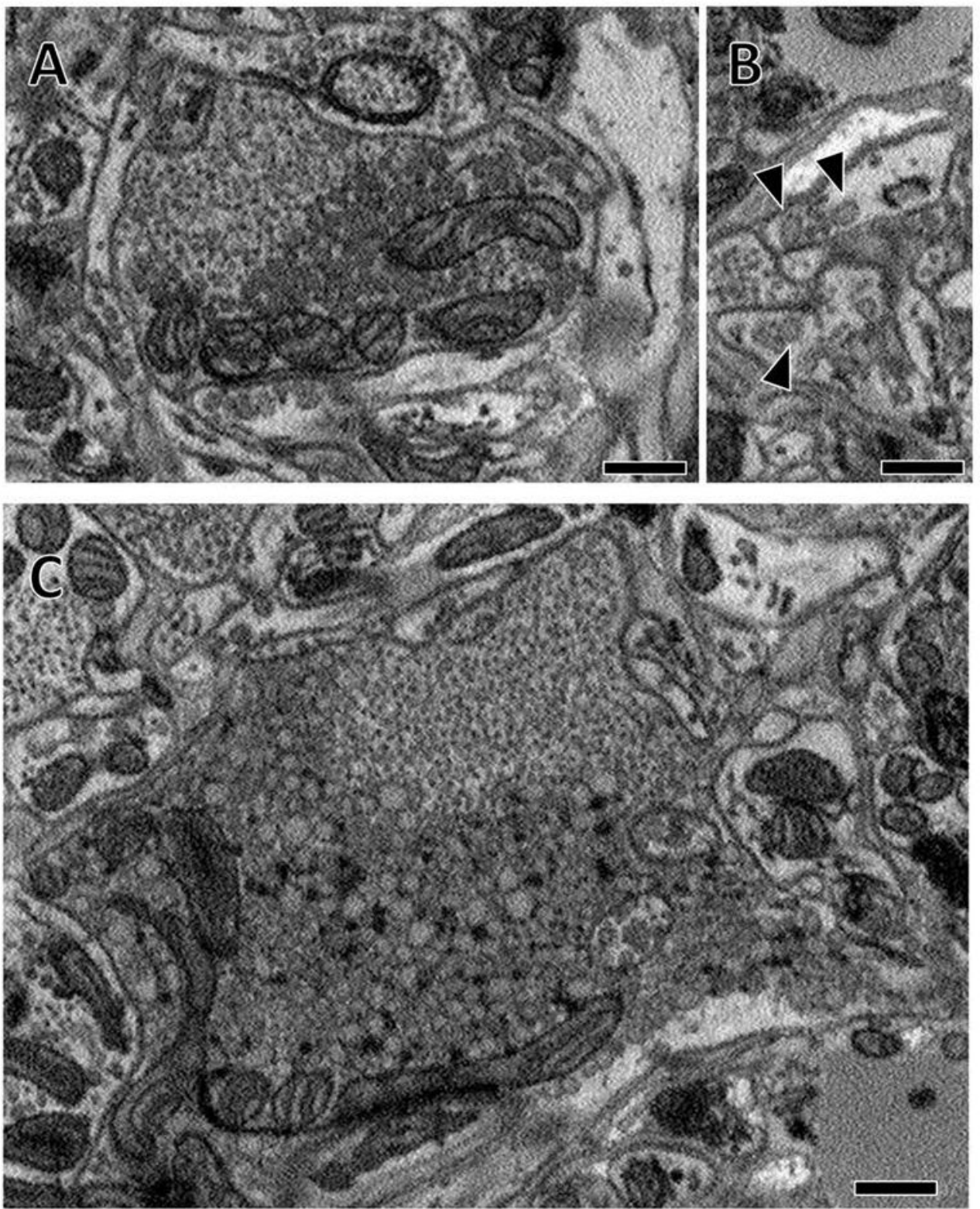

Figure 7.

Some arbors contained very large vesicles (170nm maximal diameter) of lower electron density. (A) An example bouton with a population of small synaptic vesicles surrounded by an aggregation of large low density vesicles. (B) Similar to the intensely stained dense core vesicles depicted in Figure $6 \mathrm{C}-\mathrm{D}$, these large low density vesicles were seen in the thin processes between boutons (arrowheads). (C) Some boutons containing these large vesicles were very large. Dark glycogen particles are scattered amongst these large vesicles (white arrows). Scale bars $500 \mathrm{~nm}$. 


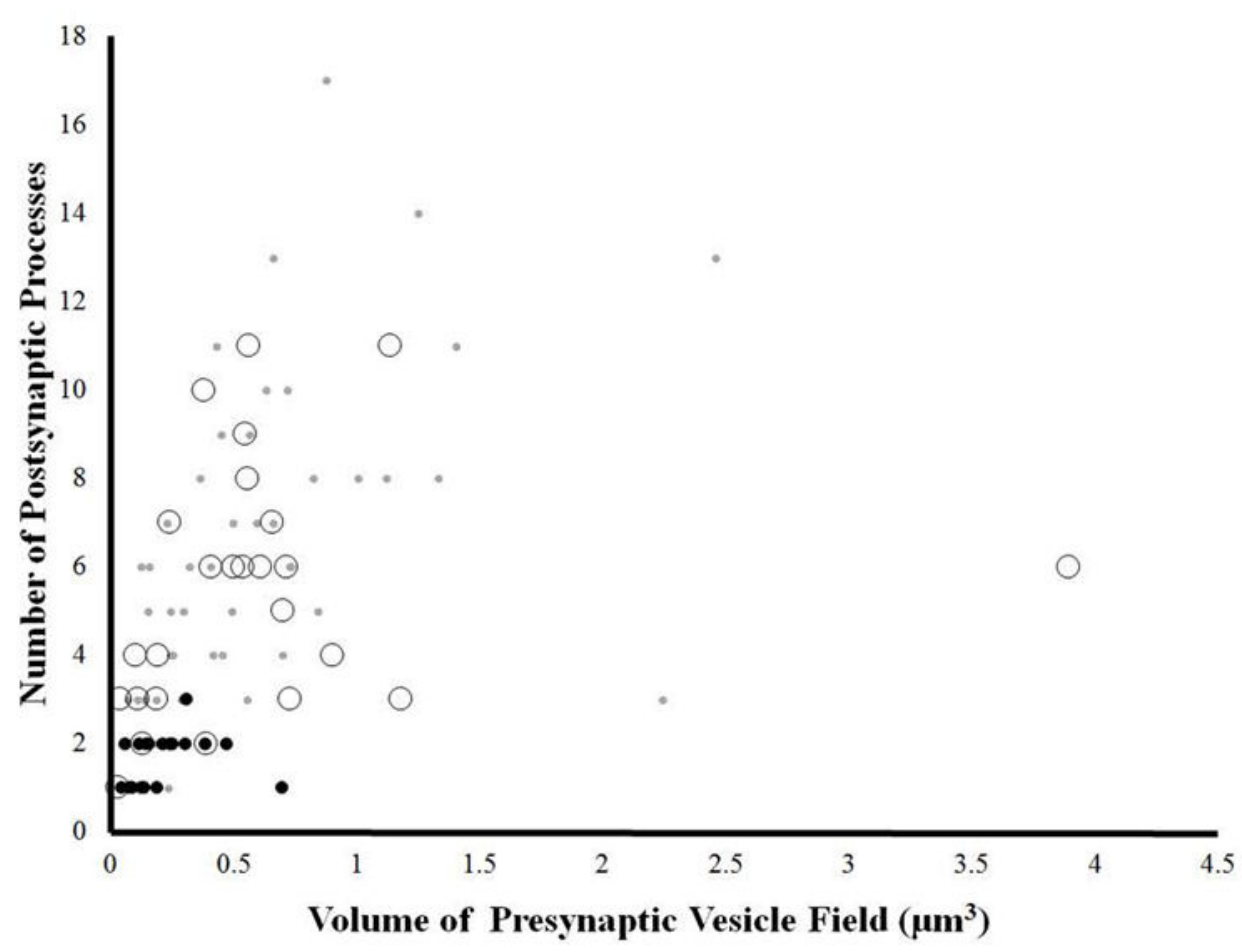

Figure 8.

The relationship between the volume of presynaptic vesicle fields and number postsynaptic contacts at individual boutons. The volume is calculated by multiplying the cumulative imaged area of the vesicle field across successive sections by the section thickness $(70 \mathrm{~nm})$. Grey dots indicate that the bouton contained primarily small presynaptic vesicles; black dots indicate that the bouton contained both small presynaptic vesicles and large intensely stained dense core vesicles; open circles indicate that the bouton contained both small presynaptic vesicles and large low density vesicles. At all data points only the volume of the small presynaptic vesicle field was calculated. 


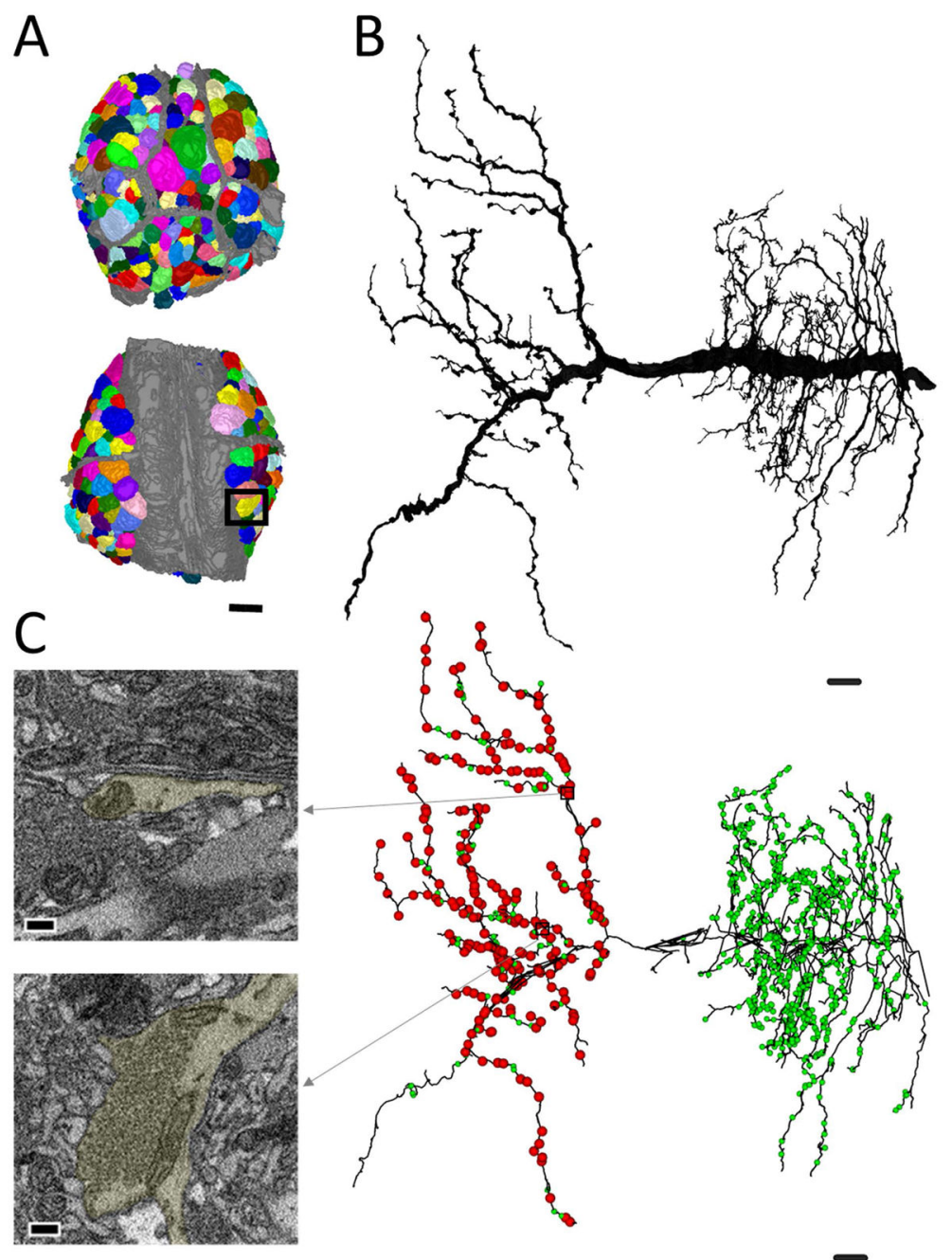

Figure 9.

The somata of some cells in the juvenile ganglion contained aggregations of intensely stained dense core vesicles or larger low density vesicles. (A) Image of a portion of the soma of an intensely-stained dense core vesicle containing cell, dense core vesicles indicated by arrows. (B) Image of a portion of the soma of large low density vesicle containing cell. (C) Map of the ganglion with blue cells indicating the somata of neurons that contained intensely stained dense core vesicles, and red cells those that contained large low density vesicles. Tan somata contained neither and were devoid of any aggregations of vesicles. The neuropilar sheath is depicted in grey. Outlined in blue box is the cell shown in (A) and outlined in the red box is the cell shown in (B). Scale bars $500 \mathrm{~nm}$ in (A) and (B), $40 \mu \mathrm{m}$ in (C). 

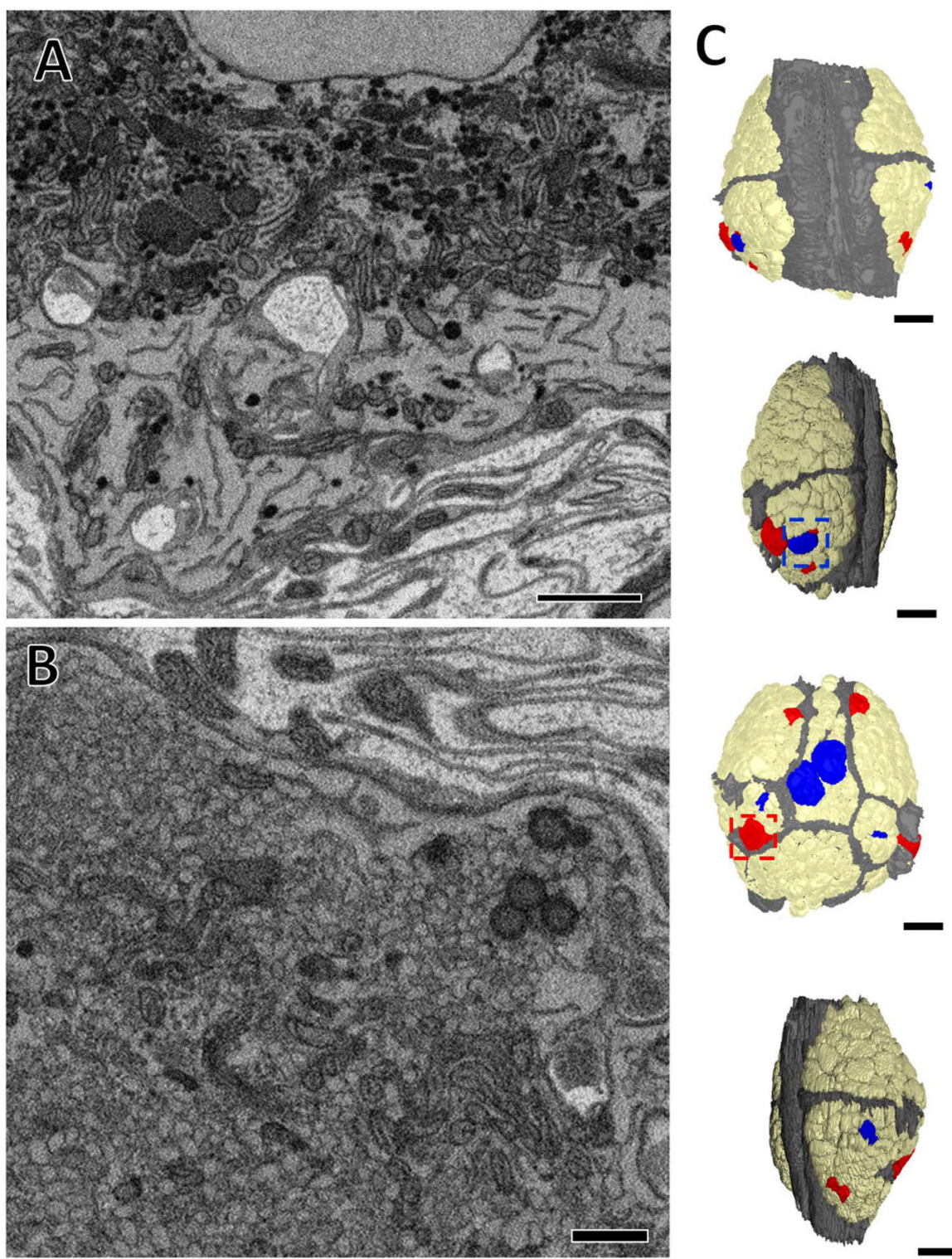

Figure 10.

A map of the entire ganglion can be used to identify, trace, and annotate full arbors. (A) A reconstruction of each neuronal soma in the ganglion (various colors) overlaid on the grey neuropilar and packet boundaries. (B) The fully reconstructed arbor of the right cell DI-1 (its soma is boxed in (A)). (C) Examples of a site of synaptic input and output and their location among all the synaptic inputs (green balls) and outputs (red balls) in the skeletonized arbor of the right cell DI-1. Note that outputs are lateralized to the contralateral half of its arbor, while inputs are distributed throughout either side. Scale bars $40 \mu \mathrm{m}$ in (A), $10 \mu \mathrm{m}$ in (B, C), $300 \mathrm{~nm}$ in insets in (C). 

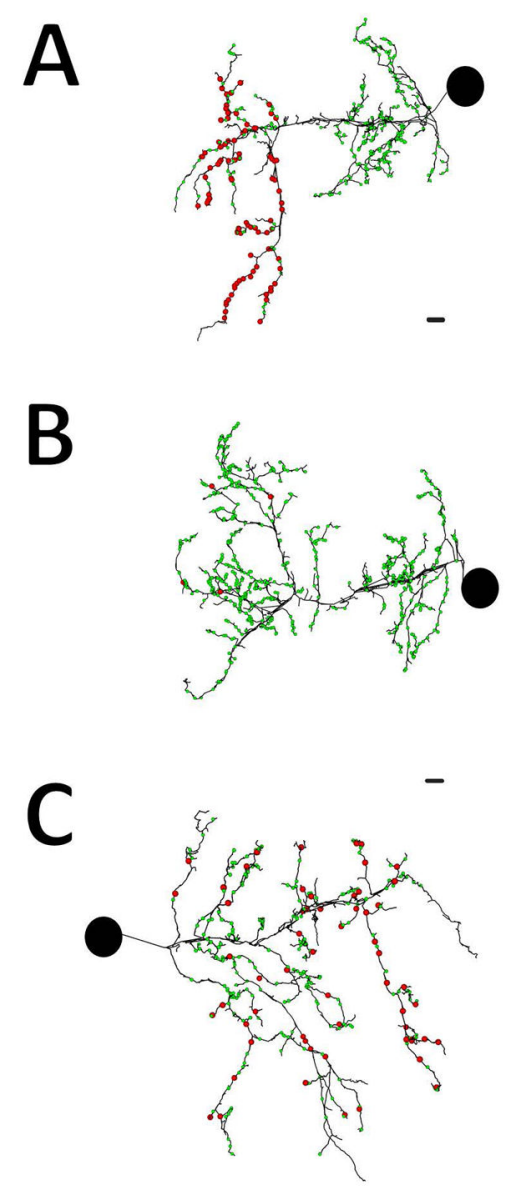

D

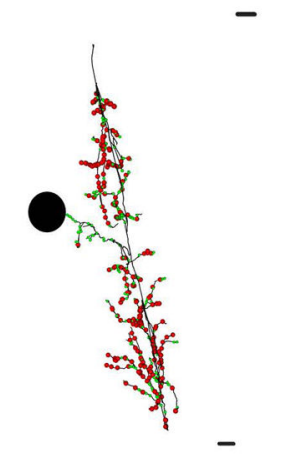

Figure 11.

Distributions of input and output sites in four neurons. In all panels green balls represent input sites and red balls represent output sites. Large black circles indicate the relative position of the somata. (A) The skeleton arbor of the inhibitory right DI-102 reveals outputs are lateralized to the contralateral half of its arbor while input sites are distributed throughout. (B) The skeleton of the excitatory right DE-3 is almost completely devoid of output sites within the ganglion with the exception of a few in the contralateral portion of its arbor. (C) The skeleton of the inhibitory left cell 116 contains input and output sites 
intermingled throughout all branches of its arbor. (D) The excitatory S cell also has input and output sites on every branch of its arbor. Scale bars $10 \mu \mathrm{m}$. 


\section{Table 1}

List of nodes, inputs and outputs per fully-traced cell in the juvenile ganglion image volume. Vesiclecontaining nodes are sites of synaptic output while synaptic input nodes are sites of input. Only one node at a presynaptic bouton was marked as "vesicle-containing". Total nodes refers to every node belonging to the skeleton of that arbor

\begin{tabular}{|c|c|c|c|}
\hline Cell Name & Total Nodes & Vesicle-containing Nodes & Synaptic Input Nodes \\
\hline S Cell & 13802 & 232 & 374 \\
\hline Left Coupling Cell & 17298 & 26 & 837 \\
\hline Right Coupling Cell & 8659 & 28 & 428 \\
\hline Right DI-102 & 18978 & 95 & 361 \\
\hline Left DI-102 & 22669 & 101 & 511 \\
\hline Right 116 & 27507 & 76 & 415 \\
\hline Left 116 & 22787 & 63 & 286 \\
\hline Left VE-4 & 20363 & 0 & 610 \\
\hline Right VE-4 & 15202 & 0 & 321 \\
\hline Left DI-1 & 29590 & 90 & 577 \\
\hline Right DI-1 & 39928 & 261 & 912 \\
\hline Left VI-2 & 16092 & 59 & 370 \\
\hline Right VI-2 & 17191 & 74 & 402 \\
\hline Right DE-3 & 21608 & 6 & 650 \\
\hline Left DE-3 & 20161 & 1 & 437 \\
\hline
\end{tabular}

